\title{
The State of Affairs in Post-Exposure Leprosy Prevention: A Descriptive Meta-Analysis on Immuno- and Chemo-Prophylaxis
}

This article was published in the following Dove Press journal: Research and Reports in Tropical Medicine

\author{
Anne Schoenmakers' \\ Liesbeth Mieras (D) \\ Teky Budiawan ${ }^{2}$ \\ Wim $\mathrm{H}$ van Brakel (ID)
}

'NLR, Amsterdam, The Netherlands; ${ }^{2} \mathrm{NLR}$, Jakarta, Indonesia
Correspondence: Anne Schoenmakers Email a.schoenmakers@nlrinternational.org
Objective: Annually, over 200,000 people are diagnosed with leprosy, also called Hansen's disease. This number has been relatively stable over the past years. Progress has been made in the fields of chemoprophylaxis and immunoprophylaxis to prevent leprosy, with a primary focus on close contacts of patients. In this descriptive meta-analysis, we summarize the evidence and identify knowledge gaps regarding post-exposure prophylaxis against leprosy. Methods: A systematic literature search according to the Preferred Reporting Items for Systematic Reviews and Meta-Analyses (PRISMA) methodology was conducted by searching the medical scientific databases Cochrane, Embase, Pubmed/MEDLINE, Research Gate, Scopus and Web of Science on Jan. 22, 2020, using a combination of synonyms for index terms in four languages: "leprosy" and "population" or "contacts" and "prevention" or "prophylaxis." Subsequently, Infolep.org and Google Scholar were searched and the "snowball method" was used to retrieve other potentially relevant literature. The found articles were screened for eligibility using predetermined inclusion and exclusion criteria.

Results: After deduplication, 1,515 articles were screened, and 125 articles were included in this descriptive meta-analysis. Immunoprophylaxis by bacillus Calmette-Guérin (BCG) vaccination is known to provide protection against leprosy. The protection it offers is higher in household contacts of leprosy patients compared with the general population and is seen to decline over time. Contact follow-up screening is important in the first period after BCG administration, as a substantial number of new leprosy patients presents three months postvaccination. Evidence for the benefit of re-vaccination is conflicting. The World Health Organization (WHO) included BCG in its Guidelines for the Diagnosis, Treatment and Prevention of Leprosy by stating that BCG at birth should be maintained in at least all leprosy high-burden regions. Literature shows that several vaccination interventions with other immunoprophylactic agents demonstrate similar or slightly less efficacy in leprosy risk reduction compared with BCG. However, most of these studies do not exclusively focus on post-exposure prophylaxis. Two vaccines are considered future candidates for leprosy prophylaxis: Mycobacterium indicus pranii $(\mathrm{MiP})$ and LepVax. For chemoprophylaxis, trials were performed with dapsone/acedapsone, rifampicin, and ROM, a combination of rifampicin, ofloxacin, and minocycline. Single-dose rifampicin is favored as post-exposure prophylaxis, abbreviated as SDR-PEP. It demonstrated a protective effect of $57 \%$ in the first two years after administration to contacts of leprosy patients. It is inexpensive, and adverse events are rare. The risk of SDR-PEP inducing rifampicin resistance is considered negligible, but continuous monitoring in accordance with WHO policies should be encouraged. The integration of contact screening and SDR-PEP administration into different leprosy control programs was found to be feasible and well accepted. Since 2018, SDR-PEP is included in the WHO Guidelines for the Diagnosis, Treatment and Prevention of Leprosy. 
Conclusion: Progress has been made in the areas of chemoprophylaxis and immunoprophylaxis to prevent leprosy in contacts of patients. Investing in vaccine studies, like LepVax and MiP, and increasing harmonization between tuberculosis (TB) and leprosy research groups is important. SDR-PEP is promising as a chemoprophylactic agent, and further implementation should be promoted. More chemoprophylaxis research is needed on: enhanced medication regimens; interventions in varying (epidemiological) settings, including focal mass drug administration (fMDA); specific approaches per contact type; combinations with screening variations and field-friendly rapid tests, if available in the future; community and health staff education; ongoing antibiotic resistance surveillance; and administering chemoprophylaxis with SDR-PEP prior to BCG administration. Additionally, both leprosy prophylactic drug registration nationally and prophylactic drug availability globally at low or no cost are important for the implementation and further upscaling of preventive measures against leprosy, such as SDR-PEP and new vaccines.

Keywords: leprosy, chemoprophylaxis, fMDA, immunoprophylaxis, MDA, prevention, post-exposure prophylaxis, SDR-PEP, rifampicin, vaccine, BCG, MiP, Mw, LepVax, M. leprae, neglected tropical diseases, NTDs

\section{Introduction}

Leprosy is a communicable disease caused by Mycobacterium leprae (M. leprae). It can result in disabilities, disfigurements, blindness, and internal organ problems. ${ }^{1}$ It is estimated that 1 million to 2 million people are living with leprosy-related disabilities, which cause severe socioeconomic consequences, including stigma and poverty. $^{2-4}$ Leprosy is one of the oldest diseases known to mankind and was once endemic on all continents. $^{5}$ Today, the disease exists primarily in resource-poor countries with often warmer climates and it is considered a neglected tropical disease (NTD). ${ }^{1,3,4,6}$ In 2018, the World Health Organization (WHO) reported a total of 208,641 new leprosy patients worldwide, a number that has been relatively stable in the past decade. $^{7}$

M. leprae was discovered, as the first bacterium that caused disease in people, by the Norwegian doctor Armauer Hansen in 1873. 5,8 Almost 150 years after Hansen's discovery, modern science still has not succeeded in growing the bacterium in vitro; it grows in humans, in nine-banded armadillos, and in immunecompromised mice. ${ }^{5,9,10}$ Both people and nine-banded armadillos are able to transmit $M$. leprae to humans. The infection mechanism of leprosy is not fully understood, but it is generally thought to spread via the respiratory route. ${ }^{1,8}$ The average incubation time of the disease is 5 years, but it can take up to 20 years before symptoms appear. $^{3}$ During this period, a leprosy patient is contagious. ${ }^{6}$ Ongoing transmission is implied by the fact that almost $10 \%$ of global new leprosy cases are children. ${ }^{7}$

Whether colonization with $M$. leprae leads to infection and disease depends on the host's resistance and genetics, as well as environmental factors. Up to $95 \%$ of people exposed to $M$. leprae do not develop the disease. ${ }^{7}$ As mentioned, most people who do develop the disease live in resource-poor settings; poor living conditions (eg, insufficient food availability, pollution, lacking health care systems and secondary chronic psychological stress) can negatively affect immune function. ${ }^{4,5,11}$ For transmission, prolonged contact with an untreated patient is considered necessary. ${ }^{1}$ The genetic and physical distance to a leprosy patient are independently associated with the risk of developing clinical disease. ${ }^{12}$ Hence, the risk for developing leprosy is increased not only in household contacts, but also in neighbors and social contacts. ${ }^{13,14}$

In the late $1940 \mathrm{~s}$, the antibiotic dapsone was the first breakthrough in leprosy treatment. The duration of treatment often lasted a lifetime, which challenged compliance and fostered dapsone resistance in the $1960 \mathrm{~s}^{3}$ When clofazimine and rifampicin were discovered in the 1950s and 1960s, these drugs were combined with dapsone, later labeled as multidrug therapy (MDT). MDT has been provided free of charge since 1995 via WHO. ${ }^{3}$ Within a few days after starting MDT, patients are no longer considered to be contagious. ${ }^{15}$

The first focus on leprosy prevention started shortly after the discovery of $M$. leprae. Compulsory isolation for persons affected by leprosy, mainly in leprosy colonies, was internationally promoted from the 19th century onward as one of the few existing prevention methods. ${ }^{16,17}$ Healthy-appearing children were frequently seperated from their parents with clinical leprosy. At the 1958 International Leprosy Congress in Japan, isolation was finally labeled as outdated and inhumane, also because dapsone as a treatment option became available. $^{16,17}$

When MDT was introduced, it was expected that early detection and prompt treatment would break the transmission chain of M. leprae. ${ }^{18}$ However, the incidence decline 
was slower than anticipated. ${ }^{19,20}$ This implies that there could be an accumulation of people with leprosy in communities who remain undiagnosed and/or untreated. ${ }^{21}$ These "missing millions" contribute to further transmission of the disease. ${ }^{21}$ Consequently, in the Global Leprosy Strategy 2016-2020, WHO determined that early case detection and targeted active case finding among highrisk groups are key to control leprosy and avert disabilities. ${ }^{22}$ At the same time, intensified populationbased approaches for case detection are no longer considered cost-effective in all contexts. ${ }^{21,23,24}$ The risk of leprosy exposure in general populations is usually low, and, as stated, geographically closer contacts of patients are more likely to develop the disease. ${ }^{12-14}$ Therefore, more targeted prevention methods were considered necessary to stop transmission. ${ }^{24}$

Over the past years, progress has been made in the fields of chemoprophylaxis and immunoprophylaxis to prevent leprosy, with a primary focus on close contacts of patients. ${ }^{23}$ In this article, we present a descriptive metaanalysis on leprosy post-exposure prophylaxis.

\section{Methods}

\section{Search}

The aim of this review is to summarize the evidence on post-exposure prophylaxis for leprosy and to identify knowledge gaps and topics for future studies. A descriptive meta-analysis method was chosen. ${ }^{25}$

Table I Search Strategy Performed on January 22, 2020

\begin{tabular}{|c|c|c|}
\hline General Syntax & Database & $\begin{array}{l}\text { Number } \\
\text { of } \\
\text { Articles }\end{array}$ \\
\hline $\begin{array}{l}\text { (leprosy OR leprae OR lepra } \\
\text { OR hansen OR hansens) AND } \\
\text { (population OR populations } \\
\text { OR persons OR contacts OR } \\
\text { people OR inhabitants OR } \\
\text { community OR communities } \\
\text { OR members) AND (prevent* } \\
\text { OR prophylaxis OR } \\
\text { prophylactic OR } \\
\text { chemoprevention OR } \\
\text { chemoprophylaxis OR } \\
\text { immunoprevention OR } \\
\text { immunopreventive OR } \\
\text { immunoprophylaxis OR } \\
\text { immunoprophylactic) }\end{array}$ & $\begin{array}{l}\text { Cochrane (title, } \\
\text { abstract, keywords) } \\
\text { Embase (title, } \\
\text { abstract, author } \\
\text { keywords) } \\
\text { PubMed/MEDLINE } \\
\text { (title, abstract, MESH } \\
\text { terms) } \\
\text { Research Gate } \\
\text { (publications) } \\
\text { Scopus (title, } \\
\text { abstract, keywords) } \\
\text { Web of Science } \\
\text { (title) } \\
\text { TOTAL }\end{array}$ & $\begin{array}{l}468 \\
237 \\
1,005 \\
15 \\
2,374\end{array}$ \\
\hline
\end{tabular}

A systematic literature search was conducted using six electronic medical databases: Cochrane, Embase, PubMed/ MEDLINE, Research Gate, Scopus, and Web of Science (Table 1). The search was performed using a combination of synonyms of the following index terms: "leprosy" and "population" or "contacts" and "prevention" or "prophylaxis," with a query translated in English, French, Spanish, and Portuguese. When possible, the search was limited to the Title/Abstract search fields in the search engines. Medical Subject Headings (MESH) terms were added to the search query if available. In addition, an article search via Infolep.org and Google Scholar was performed. Infolep is the international knowledge center for information resources on leprosy. ${ }^{26}$ Their online database also includes "grey litrature", such as meeting reports and theses. Bibliographies of the relevant articles were screened for additional articles missing in the existing yield ("snowball method"). The articles were deduplicated, and consequently, a content review was performed, first in titles and abstracts and second in full texts. Uncertainties in the selection were discussed with a second researcher until consensus was reached.

\section{Inclusion and Exclusion Criteria}

Regarding inclusion criteria, all human studies as well as meeting reports, expert opinions, editorials, and other relevant articles were included if methods for postexposure chemoprophylaxis and post-exposure immunoprophylaxis or vaccines against leprosy were discussed. Exclusion criteria were as follow: (a) studies in languages other than English, French, Spanish, or Portuguese; (b) studies for which no full text was available; (c) studies that focused predominantly on leprosy treatment instead of prevention; (d) studies that focused solely on primary prevention interventions (eg, primary vaccination in newborns); (e) studies that solely assessed immunological status changes after vaccination without assessing clinical infection; (f) study protocol descriptions or preliminary reports of clinical trial data that were later similarly described in more complete publications; and $(\mathrm{g})$ articles in which no new information was discovered (data-saturation). Every step in the search phase was documented according to the Preferred Reporting Items for Systematic Reviews and MetaAnalyses (PRISMA) literature review methodology. ${ }^{27}$ After the search, a data and text mining process was performed. ${ }^{25}$ The authors, year of publication, and study characteristics (ie, study design, population, period, geographic area, intervention type, results) were extracted 

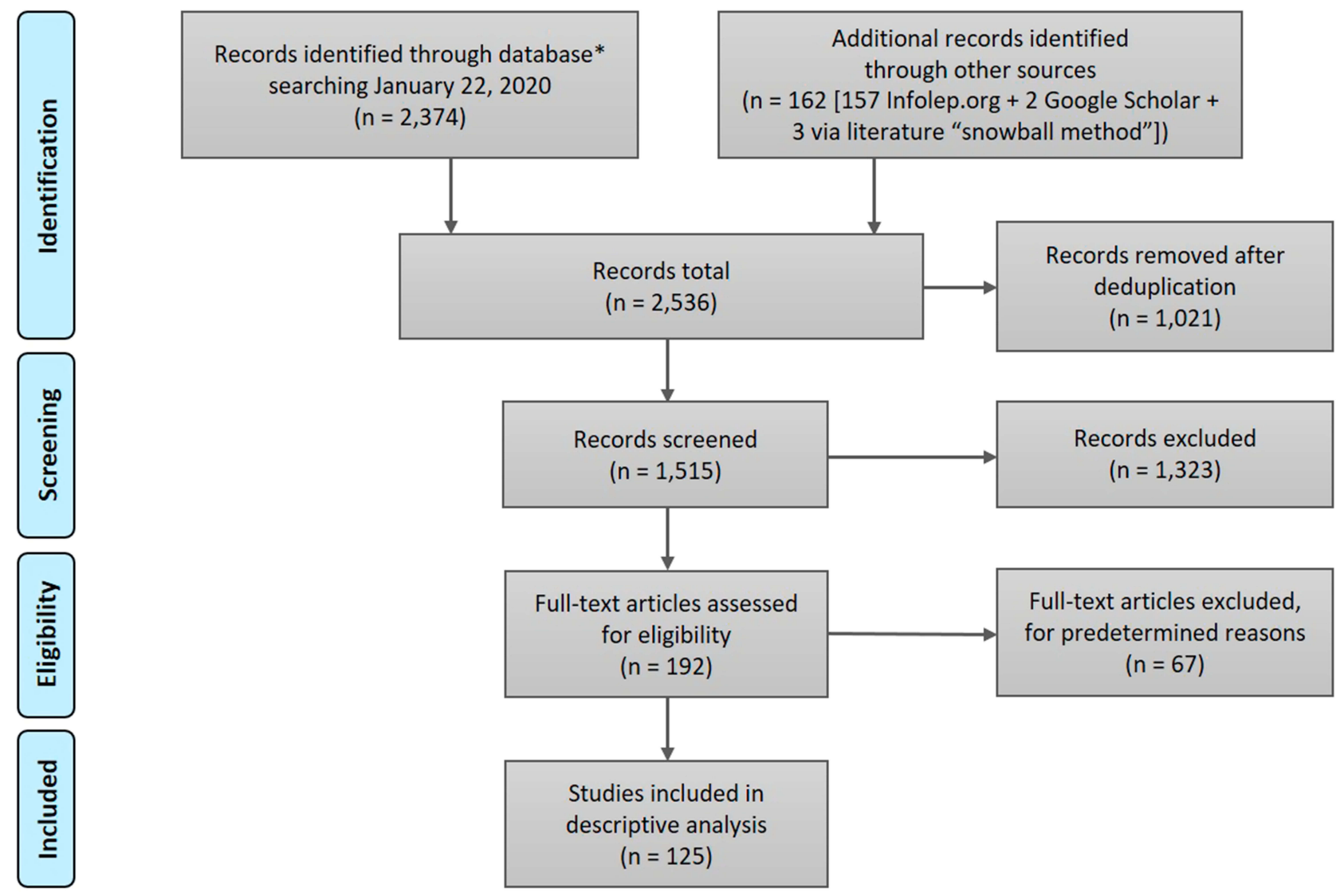

*Cochrane, Embase, Pubmed/MEDLINE, Research Gate, Scopus, Web of Science Abbreviation: $n=$ number

Figure I Preferred Reporting Items for Systematic Reviews and Meta-Analyses (PRISMA) flowchart literature search process. ${ }^{27}$

from relevant articles. The relevant information was then selected and included in this state-of-affairs literature overview.

\section{Results}

The search in the six medical literature databases on January 22, 2020 (Table 1) and the additional search via Infolep.org, Google Scholar and articles' bibliographies resulted in 2,536 articles after deduplication (Figure 1). A total of 125 articles included relevant information for this descriptive review on leprosy post-exposure prophylaxis.

\section{Immunoprophylaxis}

\section{BCG}

The bacillus Calmette-Guérin (BCG) vaccine is a live attenuated vaccine prepared from a strain of Mycobacterium bovis, derived from a tuberculous cow. ${ }^{28,29}$ The first BCG vaccine, originally developed against tuberculosis (TB), was produced by Albert Calmette and Camille Guérin in 1924 at the Pasteur Institute. ${ }^{30,31}$ The influence of BCG on the lepromin reaction, described by José Fernández in 1939, indicated that BCG might also provide leprosy protection. ${ }^{32,33}$ Currently, over 10 substrains of BCG are being manufactured. ${ }^{29}$ Approximately 100 million newborns each year receive the BCG vaccine, mainly as primary protection against TB. BCG is contraindicated in immunocompromised persons and pregnant women. ${ }^{29}$ About $95 \%$ of the people who receive the $\mathrm{BCG}$ vaccine experience a reaction at the injection site, often leaving a superficial scar. ${ }^{29,34}$

The protective effect against leprosy of BCG, usually given as primary TB prevention to newborns, varies widely in different countries. ${ }^{35-37}$ Observational studies demonstrated a larger protective effect against leprosy, possibly caused by the shorter follow-up periods, compared with experimental trials (60\% versus $41 \%) .{ }^{37}$ Long follow-up periods are needed because of the long 
incubation period of leprosy. The meta-analysis of Merle et al in 2010 stated that primary BCG vaccination in newborns is effective in reducing the risk of leprosy by $55 \%$ (95\% CI 42-67). The over-all protection of BCG vaccination against leprosy in this analysis ranged from $20 \%$ to $90 \%{ }^{37}$ Similarly to $\mathrm{TB}$, the protection by BCG against leprosy is most evident in children. ${ }^{38,39}$ WHO's Guidelines for the Diagnosis, Treatment and Prevention state that BCG at birth should therefore be maintained in at least all leprosy high-burden regions. ${ }^{7}$ The heterogeneity in the protective effect is thought to have multiple causes, including the use of different BCG vaccine strains and batches; varying study populations (eg, immunogenic characteristics, age groups); different time periods and follow-up schedules; varying geographical settings regarding environmental mycobacteria variations and M. tuberculosis; differences in leprosy burden; variations in clinical experience of health staff; and discrepancies in study methodology. ${ }^{23,29,35,37,38,40-43}$ Additionally, the protective effect of BCG in leprosy may decrease over time. ${ }^{40}$ When focusing on BCG as a post-exposure immunoprophylactic agent for leprosy contacts, the number of studies is relatively limited. ${ }^{37,43-48}$

In 1981, Stanley et al described a randomized controlled trial in which 16,150 Ugandan children, who were contacts of leprosy patients, were included between 1960 and 1964 (Table 2) ${ }^{47}$ The children did not show clinical symptoms and scored negative or weakly positive at the tuberculin test. One group received BCG vaccination; the control group was not vaccinated. The children were monitored for up to four times, over an average period of eight years. In the BCG vaccinated group, 41 children developed leprosy; in the control group, 201 developed the disease. The protective effect against leprosy was $80 \%$ (95\% CI 72-86) and did not depend upon vaccination age, gender, or grades of physical contact and genetic relationship with a patient. A small decline in protection seemed to appear at eight years follow-up, with a leprosy incidence reduction of $64 \%{ }^{47}$

Lwin et al published a randomized controlled trial performed in Myanmar (Burma) in 1985 (Table 2). ${ }^{43}$ A group of 13,066 children aged $0-14$ years received BCG; 1,531 (11.7\%) of these children were household contacts of leprosy patients. The control group counted 13,176 children, including 1,493 (11.3\%) household contacts. The inclusion period ranged from 1964 to 1968 . Annual follow-up assessments were performed until 1978-1979. The overall protective effect of BCG was
$20.4 \%$ (95\% CI 12-28). The leprosy incidence in the vaccinated group was $0.41 \%$ for females and $0.46 \%$ for males, compared to $0.48 \%$ and $0.63 \%$ for controls, respectively. A higher protective effect was found in younger children. BCG protection showed to be independent of the initial tuberculin status. A protective effect of $31.3 \%$ was seen in contacts of lepromatous/borderline patients, of $27.7 \%$ in contacts of patients with other leprosy forms, and of $18.7 \%$ among non-contacts. Weaknesses in this study are that two strains with varying bacillary count were used, resulting in varying protection rates. In addition, the described total number of contacts in this study was relatively low, and the number of follow-up moments varied (11-14 times). ${ }^{43}$ Furthermore, non-contacts may have become contacts during follow-up.

In Brazil in 2008, Düppre et al published a cohort study on the effectiveness of BCG vaccination among contacts of 1,161 leprosy patients between 1987 and 2006 (Table 2).$^{48}$ Of the 3,536 leprosy patient contacts who received BCG vaccination, 2,337 people already had a BCG scar, suggesting previous vaccination. The unvaccinated group counted 1,810 contacts, of whom 1,087 already had a BCG scar. The protection against leprosy from the BCG post-exposure vaccination was found to be $56 \%$ (95\% CI $31-71)$. For those without a primary scar, protection reached 59\% (95\% CI 27-77); for those with a primary scar, protection was $50 \%(95 \%$ CI $11-72)$. It was stated that BCG protection in contacts was not substantially affected by previous BCG vaccination. The study strongly supported the routine BCG vaccination of leprosy contacts regardless of previous vaccination. Remarkably, during the first $2-10$ months, 28 new leprosy cases - 21 vaccinated (16 without a primary BCG scar) and seven unvaccinated-were detected. Tuberculoid forms predominated. Of these new cases, 25 (90\%) had a multibacillary leprosy index case in their family. However, misclassification of contact type may have led to residual confounding. Furthermore, this study stated that no solid reason exists to doubt the disease classification data and BCG exposure, while this is debatable when examining other studies. ${ }^{29,37,48}$ Moreover, this study assumed that the contacts who did not return after the initial examination were healthy, which may not always have been the case. ${ }^{48}$

Merle et al found that BCG efficacy in studies focusing on household contacts was significantly higher compared with studies targeting the general population $(66 \%$ and $53 \%$, respectively). ${ }^{37}$ But it was mentioned that the risk of 


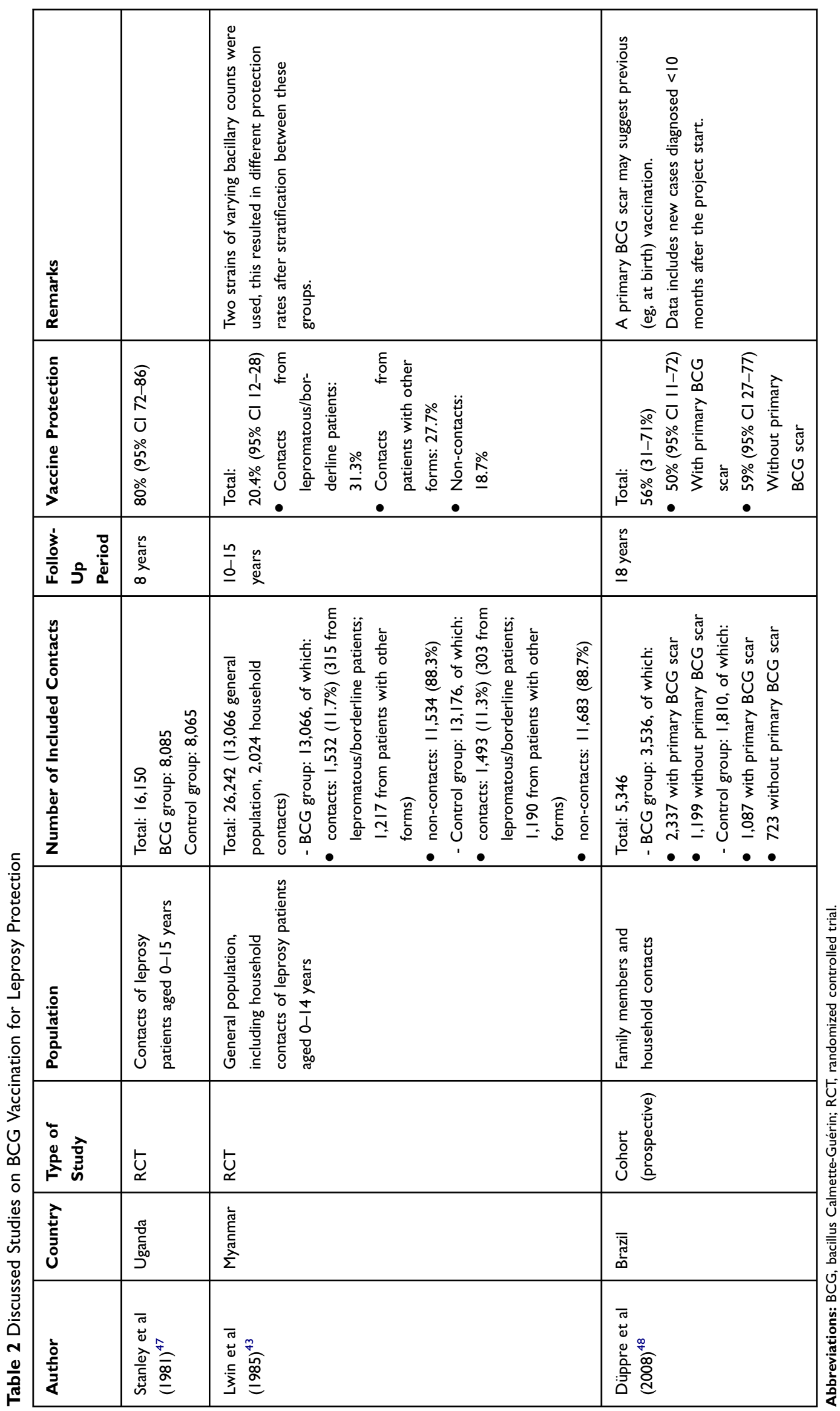


misclassification of cases and controls should be considered given the long incubation time of leprosy. Included studies were found to be heterogeneous, caused by eg, varying study designs and some study groups receiving multiple doses of BCG. Studies including BCG revaccination only or studies that did not (also) estimate the efficacy of one post-exposure dose of the BCG vaccine were not included in this metaanalysis.

\section{Other Immunoprophylactic Agents}

Literature states that several vaccination interventions show similar or slightly less efficacy in reducing the risk of leprosy compared to BCG, but few studies focus primarily on post-exposure prophylaxis. ${ }^{23,40,49-52}$ The BCG vaccine combined with heat-killed $M$. leprae had no additional effect compared with the use of BCG alone in trials in Venezuela and Malawi. The trial in Venezuela focused on household contacts of patients; the trial in Malawi included participants from a leprosy endemic population in the Karonga District. ${ }^{53,54}$ A large two-and-a-half-year double-blind controlled trial performed by Gupte et al, starting in 1991 in South India, included 171,400 people, who were not specifically described as contacts of leprosy patients, to test four different vaccines in comparison with placebo: BCG; BCG plus heat-killed M. leprae; the Indian Cancer Research Center (ICRC) vaccine, which is an $M$. leprae-related cultivable mycobacterium; and Mycobacterium $w(M w) .{ }^{51}$ A first survey shortly after the intake period suggested a non-significant negative protective effect, most dominant in BCG, that disappeared at the second survey moment. It was thought that this was caused by a combination of missed prevalent cases and new cases. After the second survey at six years, BCG plus killed $M$. leprae provided $64.0 \%$ protection (CI 50.4 73.9), ICRC gave $65.5 \%$ protection (CI 48.0-77.0), $\mathrm{Mw}$ provided $25.7 \%$ protection (CI 1.9-43.8), and BCG gave $34.1 \%$ protection (CI 13.5-49.8). ${ }^{51}$ The reason for the better protection of BCG plus killed $M$. leprae versus BCG alone in this trial, compared with the Venezuela and Malawi trials, is unclear. ${ }^{4,51,53,54}$ The described vaccine candidates were found to be safe for human use. $^{49,51,53,54}$

Sharma et al performed a double-blind trial on the $M w$ vaccine in India in the 1990s that did include household contacts of leprosy patients. ${ }^{50}$ When contacts only were vaccinated, the $M w$ vaccine showed a protective efficacy of $68.6 \%$ at the end of the 3-year follow-up period, $59 \%$ at 6 years, and $39.3 \%$ at 9 years follow-up. When both patients and contacts received the $M w$ vaccine, the observed protective efficacy was $68 \%$ at 3 years, $60 \%$ at 6 years, and $28 \%$ at 9 years, with significance found at the first two survey moments $(p<0.00005)$ and in a lesser degree at the third survey moment $(p<0.01) .{ }^{50}$ However, this study stated that early post-vaccination cases detected within 1 year of administering the vaccine were not included in the analysis, as these vaccine recipients were thought to be harboring the infection. This is a major limitation and makes the results of this trial difficult to interpret.

Truoc et al published a study on BCG alone, BCG plus $10^{7}$ killed Mycobacterium vaccae (M. vaccae or $\left.M v\right)$, and $10^{8}$ killed $M v$ alone. The study included young people, 3-20 years old, living in close contact with leprosy patients in 1988 in Ho Chi Minh City, Vietnam. ${ }^{55}$ At least twice a year, signs of leprosy were routinely sought. The study was non-randomized, and intervention allocation in this study was also doubtful: for example, 74 children who did not attend the initial examination moment were chosen as the control group. ${ }^{37,55}$ Over the entire 8 years, 14 of 74 (18.9\%) unvaccinated controls and 25 of $343(7.3 \%)$ vaccinated children developed leprosy ( $p<0.001$ ), showing a protective effect of $61.4 \%$ without significant differences among the three vaccines. The small sample size and uncertainties in scar-reading could potentially further have influenced the outcome of this study. ${ }^{29,37,55}$

\section{Promising Vaccines}

At the moment, besides $\mathrm{BCG}$, two vaccines are considered potential candidates for leprosy prophylaxis: the $M w$ vaccine, developed in India, and LepVax, developed in the United States. $^{56-60}$

Because of the described positive protective efficacy of $M w$, this cultivable, non-pathogenic mycobacterium was selected for further development. It has been sequenced and is now named Mycobacterium indicus pranii $(M i P)$ to avoid confusion with $M$. tuberculosis- $W^{61,62} M i P$ expedites bacterial clearance and shortens the recovery time in leprosy patients. $^{61-63}$ Furthermore, the addition of MiP vaccine as an immunomodulator to MDT in leprosy patients leads to speedier attainment of slit-skin smear test negativity, and it seems to have a positive effect on leprosy reactions from six months onwards. ${ }^{60,64}$ The $M i P$ vaccine has received approval from both the Drugs Controller General of India and the American Food and Drug Administration (AFDA) and is now being manufactured as Immuvac/Cadi-05 by 
Cadila Pharmaceuticals. It also seems effective in other conditions, such as TB and warts. ${ }^{61}$

In 2002, the American Leprosy Missions (ALM) partnered with the Infectious Disease Research Institute (IDRI) in Seattle to start a leprosy vaccine development trajectory. ${ }^{65}$ LepVax was developed to provide both effective preexposure and post-exposure prophylaxis against $M$. leprae infection. ${ }^{57}$ It was shown to be safe. A decrease and/or delay of neuropathy caused by M. leprae in nine-banded armadillos was also observed, making LepVax promising as immunotherapy. ${ }^{57}$ In August 2017, the vaccine was approved by the AFDA. ${ }^{65}$ LepVax testing in humans is ongoing.

\section{Post-Exposure Chemoprophylaxis Dapsone/Acedapsone}

When dapsone was the sole treatment of choice for leprosy, people sought ways to protect contacts of leprosy patients. The idea to introduce post-exposure prophylaxis (PEP) was based on the hypothesis that close contacts of leprosy patients have already been infected by $M$. leprae by the time the patient is diagnosed and that post-exposure chemoprophylaxis would prevent the contacts from developing the clinical disease. The first reports on chemoprophylaxis trials, using dapsone, in India and Seoul were published in the 1960s. ${ }^{66-68}$ These first results suggested that weekly or biweekly doses of dapsone given to contacts of leprosy patients for a longer duration (months to years) had a protective effect against leprosy. In subsequent years, this was confirmed by additional studies in other contexts. ${ }^{69-74}$

In the 1970s, several studies were published on the use of diacetyldapsone (DADDS) or acedapsone, a long-acting repository sulphone given by injection. The administration intervals of approximately 10 weeks added to the operational feasibility; also, no toxicity was found. ${ }^{75-77}$ Neelan et al found that even a short duration of chemoprophylactic treatment with acedapsone through a series of three injections provided protection after four years of followup. $^{78}$

Smith and Smith conducted a meta-analysis in 2000 to quantify the efficacy of dapsone chemoprophylaxis against leprosy. ${ }^{79}$ They concluded that chemoprophylaxis is an effective method to reduce the leprosy incidence. They also stated that it is more cost-effective in household contacts than when administered to entire communities. They advised more research on simple, single-dose regimens. This could improve compliance and feasibility in more remote settings.

\section{Rifampicin/ROM}

In 1999, a workshop on leprosy prevention with international experts was held in the Federated States of Micronesia. ${ }^{80}$ During this workshop, studies were presented introducing new regimens comprising rifampicin alone or rifampicin combined with ofloxacin and minocycline (ROM). An important improvement of these new regimens was that they were given either as a singledose or were repeated once, as compared to dapsone that was given repeatedly for several years. The first experience with rifampicin as chemoprophylaxis was gained in 1988 in French Polynesia on the South Marquesas Islands, involving the screening of 2,786 inhabitants and administering $25 \mathrm{mg} / \mathrm{kg}$ single dose rifampicin (SDR) to 2,751 inhabitants (98.7\%) (Table 4) and also 3144 South Marquesan "emigrants" with their families. ${ }^{81,82}$ During the four-year follow-up, a decrease in the new case detection rate of $80 \%$ was observed. Against a background of an already declining incidence after MDT introduction, the intervention's efficacy was estimated to be $50 \%{ }^{82}$

In addition, experiences were reported with SDR or the regimen ROM as chemoprophylaxis, aiming at a decrease in the new case detection rate in the Federated States of Micronesia, Kiribati, and the Republic of the Marshall Islands. ${ }^{83-88}$ No serious side effects were recorded.

Some concerns about the complexity of the intervention and the costs involved in chemoprophylaxis against leprosy were discussed. ${ }^{89,90}$ A plea was made for a simple regimen to target high-risk groups, maximizing the costbenefit ratio. It was also acknowledged that more operational research was needed to better define the populations that could benefit the most. ${ }^{91}$

In the years after the workshop, more publications provided evidence on the effect of chemoprophylaxis, but it was also recognized that the studies were not placebo controlled and/or could not distinguish between the effect of the chemoprophylaxis and the effect of intensified case detection interventions. ${ }^{92,93}$ Cuba, a low endemic country, was alone in starting early with nationwide implementation of SDR-PEP in $2002 .{ }^{94}$

\section{The COLEP Study}

Moet et al conducted a large cluster-randomized controlled trial in Bangladesh, the COLEP study, to determine the effectiveness of SDR in preventing leprosy in close contacts of leprosy patients (Table 4). ${ }^{95}$ The study focused on household contacts, neighbors, and social contacts, because these contacts were identified as most at risk. ${ }^{12}$ 
Approximately 10,000 contacts received SDR, while another 10,000 received placebo. Exclusion criteria were as follow: age $<5$ years, pregnancy, liver or renal disease, signs or symptoms of leprosy or TB and previous rifampicin intake, refusal, or contacts who were residing only temporarily in that area. The study showed that SDR given to contacts of new leprosy patients reduced their risk of developing clinical leprosy by 57\% (95\% CI $33-72) .{ }^{96}$ The effect was more pronounced in more distant contacts and less in blood-related family. As part of the COLEP study, the cost-effectiveness of using SDR to prevent leprosy was assessed to be good at all contact levels. ${ }^{97}$ The incremental cost-effectiveness ratio (ICER) was US\$ 158 per one prevented leprosy patient. ${ }^{97}$ Overall, rifampicin administration to screened contacts of leprosy patients was shown to be affordable and feasible. ${ }^{95,97}$ An additional post hoc finding during the COLEP study was that rifampicin given to contacts who have had a childhood BCG vaccination, had a protective effect of $80 \%$ (95\% CI 50-92). ${ }^{98}$ In another COLEP substudy, the acceptability of SDR was assessed among healthy community members. ${ }^{99}$ All study participants expressed that they would be willing to take chemoprophylaxis, even after it was explained that full protection against leprosy was not guaranteed. However, many participants also expressed that they would not like to share information on their disease status with neighbors and social contacts if they would have leprosy. ${ }^{99}$

\section{In Follow-Up of the COLEP Study}

Another workshop on the use of chemoprophylaxis against leprosy was held in 2006, in the Netherlands. ${ }^{100}$ Here, the preliminary COLEP data were presented as well as results from another controlled trial using rifampicin in two repeated doses on a group of five islands in the Flores Sea in Indonesia (Table 4). ${ }^{95,101}$ This trial showed similar results to the COLEP study, with a risk reduction of about $60 \%$ on the island where the total population was screened and received two dosages of rifampicin when eligible. ${ }^{95,101}$ Preliminary data of a randomized controlled chemoprophylaxis trial in India, the results of which were never individually published, were presented by Declercq in this workshop. ${ }^{100}$ SDR given to household contacts was compared with placebo and showed a $74 \%$ risk reduction after 4-5 years follow-up.

During the workshop, concerns were raised regarding the risk of inducing antibiotic resistance when using rifampicin on a large scale. ${ }^{100} \mathrm{Ji}$ addressed these concerns with the argument that resistance is very unlikely to emerge because SDR is proven not to select resistant mutants, as shown in publications on multibacillary patients who relapse after SDR treatment. ${ }^{89,100}$

Experts agreed that the use of a single dose as chemoprophylaxis was to be preferred, that powerful bactericidal activity was a necessity, and that adverse events risk should be minimal. Several drugs in the rifamycin and quinolone group were considered suitable candidates for leprosy chemoprophylaxis, though the experts agreed that clinical trials were needed to provide evidence of their effectiveness and that drug prices should be considered. ${ }^{95}$

The following requirements were formulated for operational research programs: (a) screening of contacts for signs and symptoms of leprosy and TB; (b) provision of chemoprophylaxis under direct supervision; (c) a recording and reporting system; (d) training of health workers; (e) proper information for people receiving chemoprophylaxis; and (f) a system for antibiotic resistance monitoring. ${ }^{95}$

In 2009, an updated meta-analysis of randomized controlled trials of leprosy chemoprophylaxis was published by Reveiz et al. ${ }^{102}$ They confirmed the review by Smith and Smith, concluding that the use of rifampicin and acedapsone/ dapsone as chemoprophylaxis can help reduce leprosy incidence and that post-exposure chemoprophylaxis should become embedded in leprosy control programs. ${ }^{79,102}$ In the same year, the WHO South-East Asia Regional Office addressed the need for further research, based on an information consultation in the United Kingdom. ${ }^{103}$

In 2010, the Technical Commission of the International Federation of Anti-Leprosy Associations (ILEP) published a Review of Leprosy Research Evidence (2002-2009) and Implications for Current Policy and Practice. ${ }^{104}$ They strongly recommended additional research on chemoprophylaxis implementation to evaluate acceptability, cost-effectiveness, feasibility, and ethical issues concerning disease disclosure and new regimens. ${ }^{104}$

After Cuba, SDR chemoprophylaxis against leprosy was introduced in Morocco in 2012, another low-endemic country. $^{105}$ There, leprosy detection declined over the years, showing an annual percentage reduction in leprosy detection rate of 16.8 (95\% CI 29.2-2.3) from 2012 to 2017 , compared with a reduction of 4.7 (95\% CI 7.32.0), between 2000 and 2012, before SDR introduction (Table 4). ${ }^{106}$ 


\section{Modeling}

To better understand the long-term effects of chemoprophylaxis on leprosy, several modeling studies have been performed using the mathematical SIMCOLEP model developed by Erasmus MC. ${ }^{107}$ Using this model, Fisher et al demonstrated that leprosy incidence would be substantially reduced in a context similar to the COLEP study location (Bangladesh) by a combination of childhood BCG and chemoprophylaxis for screened household contacts. ${ }^{107}$ De Matos et al predicted a declining new case detection rate in Para State in Brazil, combining contract screening with chemoprophylaxis. ${ }^{108}$ Mathematical modeling by Gilkison et al for Kiribati, a high leprosy burden Pacific island, also suggested that leprosy incidence can decrease when using an intensive chemoprophylaxis approach. ${ }^{109}$ The model predicted the best results for the intensive chemoprophylaxis approach that combined a household contact approach with more than one round of mass SDR in consecutive years rather than every second year. ${ }^{109}$

An expert meeting on chemoprophylaxis against leprosy in 2014 in Switzerland re-emphasized the importance of operational research on SDR-PEP. ${ }^{110}$ Some lessons learned from a pilot project implementing SDR-PEP in Sampang District, Indonesia, were presented by Dandel. He stated that SDR-PEP implementation requires: (a) ongoing support and supervision; (b) strong local ownership; (c) continuous motivation of healthcare workers; and (d) adequate loose rifampicin supply routes. Stakeholders received the intervention positively. It was also seen as an opportunity to present health education, but stigma caused index patients to hesitate in disclosing their disease status to people outside their household. Nevertheless, an average of 22 contacts per index patient were included in the first year (Budiawan, unpublished report). During the expert meeting, Mahotarn presented results of another randomized, placebo-controlled trial with SDR conducted in Thailand with a similar set-up as the COLEP study. ${ }^{95,110}$ After 5 years, the relative risk in

Table 3 Factors to Successfully Target Contacts of Leprosy Patients. Expert Meeting Switzerland, 2014

Contact screening needs to be integrated into the leprosy control program.

Community stigma needs to be addressed.

Contextualized health education messages are needed.

Funding and support must be sustainable.

Healthcare workers need to be trained and motivated to follow

program guidelines and maintain accurate records.

Note: Data from Smith et al. ${ }^{110}$ the rifampicin group was 0.48 when compared with controls. This was similar to the difference observed in the COLEP trial, though not statistically significant $(p=0.105)$ because the study was not powered to detect risk reductions below $50 \% .{ }^{95,110}$ The expert meeting identified several important factors for the success of interventions that target contacts of leprosy patients (Table 3). ${ }^{110}$

The meeting stated that high-endemic pockets would most likely benefit more from a mass drug administration (MDA) or "blanket" approach than the contactbased strategy. ${ }^{110}$ In such settings, the entire community may be considered contacts. The experts recognized that PEP implementation against leprosy should not be limited to high-endemic settings because low-endemic areas are likely to show a high clustering level. ${ }^{110}$ Richardus et al found that when the endemicity of leprosy declines, a gradually higher proportion of new patients is found amongst the contacts of known cases. ${ }^{111}$ This aligns with field observations in West Java, where leprosy endemicity varies; in the lowendemic districts, the proportion of new cases detected through contact screening is relatively high (Budiawan, unpublished report).

The need for more research on the feasibility of implementing SDR-PEP, including its cost-effectiveness and acceptability in different geographical and sociocultural environments, was recognized. ${ }^{112}$ This and the urge to learn how to operationalize this innovative intervention led to the development of the LPEP program.

\section{LPEP Program}

In 2014, a multicountry research project was developed involving ministries of health, international non-governmental organizations (NGOs), scientific institutes, leprosy experts, and a donor (Table 4). ${ }^{24,110}$ The LPEP program, as operational study, aimed to accelerate the uptake of evidence on SDR-PEP effectiveness by gathering data on the impact and feasibility of implementing SDR-PEP as part of routine leprosy control. ${ }^{24}$ The program was implemented in highendemic areas in India, Brazil, Indonesia, Myanmar, Nepal, Tanzania, and Sri Lanka. Cambodia was also inlcuded, but followed a different study protocol. ${ }^{24,117}$ Before implementation, an expert meeting was convened to address concerns regarding the risk of inducing rifampicin resistance in TB. ${ }^{113}$ The experts concluded that SDR given to contacts of leprosy patients, in the absence of symptoms of active TB, poses a negligible risk of generating resistance in $M$. tuberculosis in individuals and populations. ${ }^{113}$ 


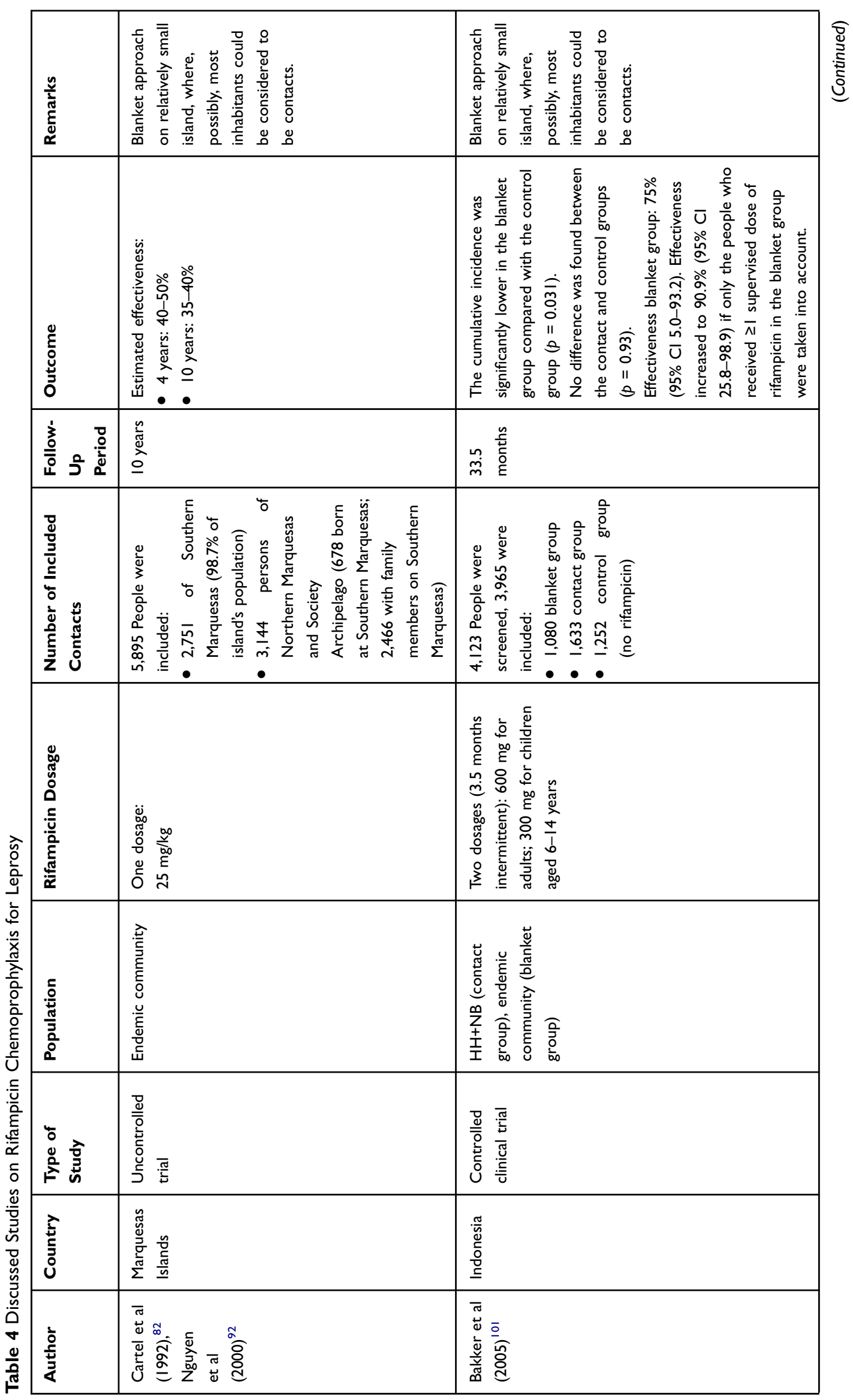




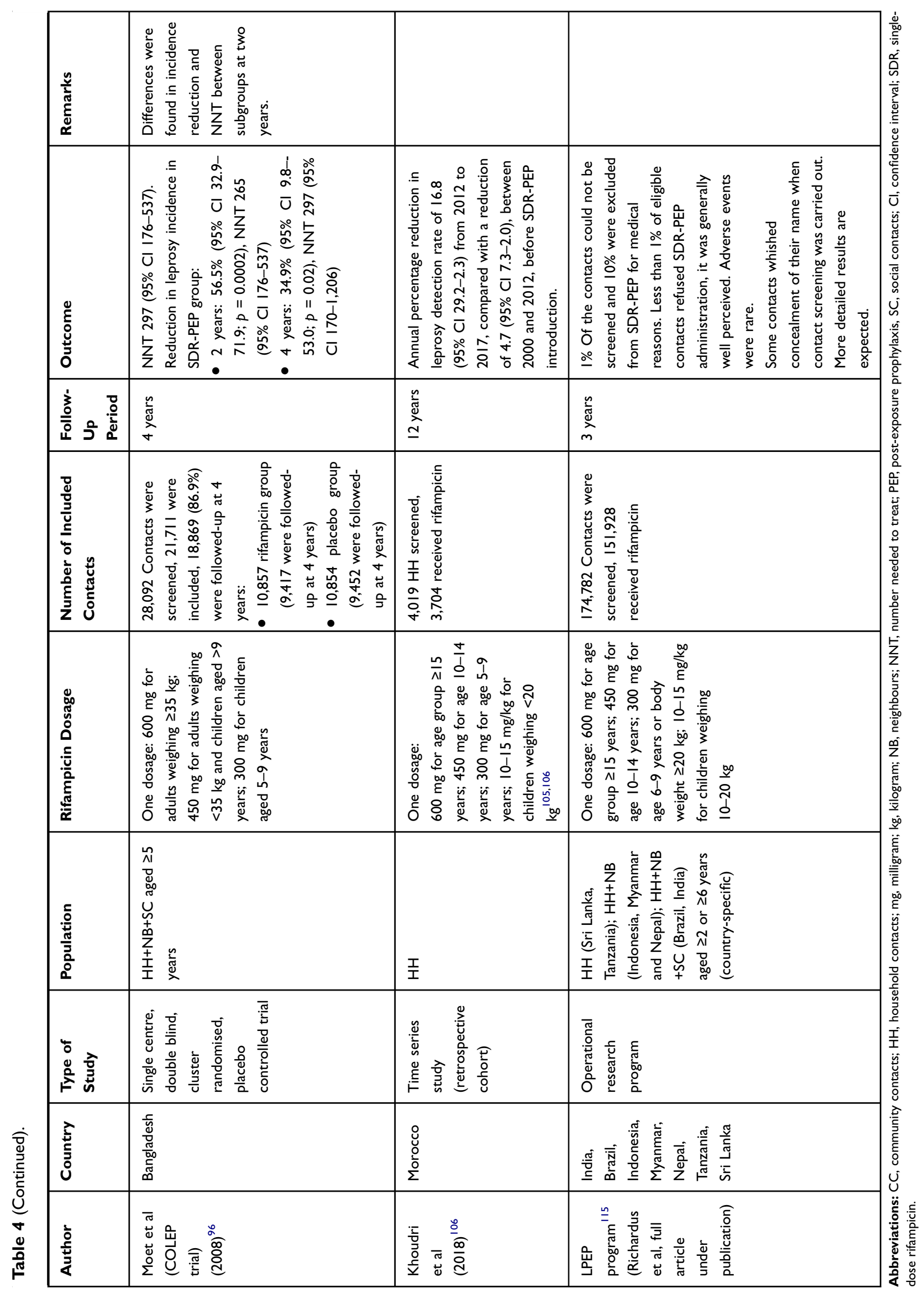


Two years into the LPEP program, the integration of contact screening and SDR-PEP administration into different leprosy control programs had proved to be feasible and well accepted by all stakeholders. ${ }^{114}$ The program was reported to have invigorated leprosy control. After three years, approximately 175,000 contacts of recently diagnosed leprosy patients had been screened. Only 10\% were not eligible for SDR-PEP, because of young age ( $<2$ years or $<5$ years, depending on the country), liver or renal disease, signs and symptoms of leprosy or TB, pregnancy, known allergy to rifampicin, rifampicin use in the past 2 years, or refusal $(<1 \%)$. SDR-PEP was found to be safe; adverse events were very rarely reported (Richardus et al, full article under publication). ${ }^{115}$ Mathematical modeling predicted that implementing SDR-PEP could potentially accelerate the reduction of new cases and, therefore, could potentially accelerate the cessation of M. leprae transmission (Blok et al, under publication). It is also estimated to be cost-effective when assessing SDR-PEP in the Indian health system (US\$ 2,873 per new leprosy case averted). ${ }^{116}$ Demonstrating the operational feasibility of integrating SDR-PEP into leprosy programs on such a large scale in multiple settings has contributed to WHO recommending SDR-PEP in its Guidelines for the Diagnosis, Treatment and Prevention of Leprosy. ${ }^{52}$

As part of the LPEP program, two specific approaches for implementing SDR-PEP were piloted. One was a blanket approach in Lingat village on Selaru Island, a remote highendemic village in Indonesia. ${ }^{116}$ The blanket approach involved total population screening in $2016(\mathrm{n}=2065)$. During two visits in consecutive years, 1,671 inhabitants were screened (88\%), and 1,499 (79\%) received SDR-PEP. During these visits, 43 new leprosy patients were diagnosed with leprosy $(2,263 / 100,000)$. During the third visit, 10 new leprosy cases in 1,481 screened persons $(484 / 100,000)$ were detected. The other approach made use of "drives" and was implemented in Cambodia, a low-endemic country. The drives in Cambodia were carried out by a mobile team with leprosy experts who screened the contacts of all patients diagnosed in the previous 10 years, and administered SDRPEP. ${ }^{117}$ In the first four operational districts, 855 contacts of 86 index patients were traced and screened, 828 (97\%) received SDR-PEP, and four new leprosy patients were identified $(468 / 100,000)$. Both the blanket approach and the drive approach required sufficient resources and thorough logistic preparation, but were operationally feasible. Regular monitoring is required to identify the long-term impact. An LPEP substudy in India, Indonesia, and Nepal assessed how the intervention changed the perception of main stakeholders regarding leprosy. ${ }^{118}$ The LPEP program was perceived positively, though more research was recommended on providing accurate and understandable health information to contacts, and on approaches that do not require disclosure of the index patient. The LPEP program also had a positive effect on people's knowledge regarding leprosy (Mieras et al, unpublished report). Another side study was an acceptability study carried out in India. ${ }^{119}$ The results of this study aligned with the results of the main study and the perception study, illustrating that contact screening and SDR-PEP distribution in Dadra and Nagar Haveli, India, were well accepted by the stakeholders. ${ }^{115,118-120}$

The lessons learned from the LPEP program led to a recommended minimal set of data required to monitor contact tracing activities and SDR-PEP administration for leprosy control in a routine setting. ${ }^{121}$ Based on the experiences gained from the LPEP program, an SDR-PEP Toolkit was developed to support national leprosy program managers concerned with the practical aspects of implementing SDR-PEP, such as advocacy, training, and reporting and recording. ${ }^{120}$

\section{Prevention as Part of Routine Leprosy Control}

In 2017, Gillini et al collected information to determine the extent of leprosy post-exposure immuno- and chemoprophylaxis in countries around the world as national policy. ${ }^{122}$ A total of 66 countries responded, representing $95 \%$ of the total reported global leprosy burden. Nationwide routine implementation of SDR-PEP was reported by only a few countries with a low burden, such as Cuba (2002), Morocco (2012), and Samoa (2015).

Since SDR-PEP is addressed in the 2018 WHO Guidelines for the Diagnosis, Treatment and Prevention of Leprosy, more countries are implementing SDR-PEP in their national leprosy control programs. ${ }^{52}$ For example, in India and Indonesia, the ministries of health adopted SDR-PEP as a main strategy in leprosy prevention in 2018 and 2019, respectively. ${ }^{144,122,123}$ This year, WHO is expected to publish a technical guidance document whith a description of the steps that could be taken for the implementation of both contact tracing and postexposure prophylaxis (WHO, under publication). The availability of preventive measures contributed to a new momentum to work toward stopping the transmission of leprosy. ${ }^{124}$ 


\section{BCG and SDR-PEP Combined}

Lwin et al concluded that BCG vaccination alone is not likely to be the solution for leprosy control. ${ }^{43}$ As described, Schuring et al performed a secondary analysis on the COLEP trial. The individual protective effect of BCG 57\% (95\% CI 24-75) and SDR-PEP 58\% (95\% CI 30-74) demonstrated a combined protective effect of $80 \%$ (95\% CI 50-92). ${ }^{98}$ After the findings of the COLEP trial, a single-center, cluster-randomized controlled trial (MALTALEP) was conducted to determine whether possible excess cases in the first year after immunoprophylaxis (with BCG) could be prevented with chemoprophylaxis (with SDR) without affecting BCG's protective effect. ${ }^{44,125}$ In the intervention group $(\mathrm{n}=7,609)$, BCG vaccination was followed by SDR after $8-12$ weeks. Controls $(n=7,379)$ received only BCG. The combined preventive outcome was expected to be long-lasting and better than the effect of solely BCG or SDR-PEP. However, it was found that one third of all new leprosy cases among contacts had appeared 8-12 weeks after BCG vaccination, before SDR-PEP was administered. This made it impossible to determine whether excess cases in the first year after immunoprophylaxis (with BCG) could be prevented with SDR-PEP without affecting the protective effect of BCG. ${ }^{126}$ This increase of cases after immunoprophylaxis administration in the first year is consistent with other studies. ${ }^{48,51,127-}$ 129 Therefore, Richardus et al concluded that BCG vaccination followed by SDR as a routine intervention is not recommended in leprosy control.

\section{Discussion}

In a time in which a new infectious disease, coronavirus disease 2019 (COVID-19), dominates the news, it is important to also keep paying attention to one of the oldest diseases known to mankind. Today, leprosy still has devastating outcomes, mainly affecting the most marginalized. Preventive methods are needed to stop the transmission of this disease.

\section{Immunoprophylaxis}

Globally, BCG vaccination is mainly used as the primary TB prevention method in newborns. As stated, in its 2018 Guidelines for the Diagnosis, Treatment and Prevention of Leprosy, WHO recommends only that BCG at birth should be maintained in at least all leprosy high-burden regions. ${ }^{7}$ Nevertheless, since the early 1990s, the Brazilian Ministry of Health has recommended intradermal BCG for household contacts of leprosy patients. ${ }^{46,130}$ Contacts with no or one primary scar receive BCG; contacts with two BCG scars do not receive another $\mathrm{BCG}$ dosage. ${ }^{46,131}$ However, scar reading is not fully reliable, and scar formation does not always occur. ${ }^{29,37}$ For example, $16-24 \%$ of BCG vaccinated individuals do not develop a scar. ${ }^{34}$ In infants vaccinated below the age of 1 month, which is common when BCG vaccination is routinely given to newborns (eg, in Brazil), fewer than $80 \%$ have a recognizable scar at the age of $4 .{ }^{132}$ Furthermore, the evidence regarding the effectiveness of BCG revaccination is conflicting, because of the wide variation in study outcomes. ${ }^{23,46,52,54,128,133,134}$

When assessing other immunoprophylactic agents, BCG plus heat-killed M. leprae was found not to have an additional effect in leprosy prevention when compared with BCG alone. ${ }^{51,53,54}$ Even if BCG plus heat-killed M. leprae provided additional protection, further development of a vaccine containing killed $M$. leprae is challenging because mass production would need to occur in armadillos or in immune-compromised mice. ${ }^{49}$ Studies on the ICRC and $M$. vaccae vaccines were limited, rarely focused on solely post-exposure prophylaxis, and were often of debatable quality. It is therefore not entirely clear if these two immunoprophylactic agents should be fully ruled out when trying to prevent leprosy transmission.

More research is also needed on newly developed vaccines like LepVax as well as on the vaccine $M i P$ $(M w)$. Furthermore, the introduction of new TB vaccines, possibly replacing BCG, could have a serious impact on leprosy. The number of vaccine studies is much greater in the TB world, consistent with the incidence numbers, compared with the leprosy research field. ${ }^{56}$ Despite the possibility of cross-protection between the two diseases, the potential impact on leprosy by TB vaccine candidates is rarely considered in TB research. ${ }^{49,56}$ Only two recombinant subunit TB vaccines (ID83/GLA-SE and ID93/ GLA-SE) have also been laboratory tested for their potential use against leprosy. ${ }^{56,135}$ More integration and harmonization between the TB and leprosy vaccine research groups would therefore be valuable. ${ }^{56}$

Additionally, after the administration of BCG and other immunoprophylactic agents for leprosy prevention, a relative increase in the number of new (paucibacillary) leprosy patients is seen in the first follow-up year. ${ }^{4,51,127-129}$ A possible explanation for this is that BCG is catalyzing the existing anti-mycobacterial immunity in people infected with M. leprae, resulting in the clinical appearance of tuberculoid leprosy after BCG vaccination. ${ }^{48,127}$ Gormus and 
Meyers described the reasoning for the doses chosen in trials with integral mycobacterial vaccines as arbitrary. ${ }^{129}$ They argue that dosages may be too high, resulting in the protection of some, but also resulting in increased susceptibility to leprosy in other individuals shortly after vaccination. An individual's prior exposure to environmental mycobacteria may also affect the outcome of mycobacterial vaccines. ${ }^{43}$ Additional research with varying dosages and in different target groups would be recommended.

Richardus et al concluded that BCG vaccination followed by SDR as a routine intervention is not recommended in leprosy control. ${ }^{46}$ The MALTALEP study was challenged in finding significance because of insufficient statistical power. ${ }^{44,125}$ A well-powered study focusing on the reversedMALTALEP order (first SDR administration, followed by BCG) would therefore be of great value.

Considering the above results, contact screening followup is especially important in the first period after BCG administration, as a relatively large number of new patients presents three months post-vaccination. ${ }^{48,51,127-129}$ This may also count for other immunoprophylactic agents.

As discussed, many immunoprophylaxis studies lacked power. More well-designed, sufficiently powered and long-lasting (regarding the leprosy incubation period) studies in this field are therefore suggested.

But, given the evidence for the effectiveness of SDRPEP and the WHO guidelines for its use, the ethics of testing new post-exposure immunoprophylactic approaches for leprosy prevention without combining them with chemoprophylaxis in both the intervention and control group needs to be discussed. $96,98,115,126,136$

\section{Chemoprophylaxis}

The promising findings of the COLEP study, the systematic reviews, and the 2007 workshop recommendation on additional, operational research were not immediately pursued. This was partly because the use of SDR-PEP has been criticized. ${ }^{137-140}$ Concerns have been raised about the fact that it does not provide long-term protection like immunoprophylaxis, and that the COLEP study revealed that it provides an overall risk reduction of $57 \%$ and only $24 \%$ in blood-related household contacts, though the latter finding was not significant $(p=0.49)$ because the study was not powered for subgroup analysis. ${ }^{112,138,139}$ However, the effect seen in all contact subgroups trended with the overall risk reduction of $57 \%{ }^{112}$ Other criticism concerned the logistics and cost-effectiveness of the intervention. ${ }^{138}$ Though, studies have demonstrated that both contact examination and SDR-
PEP implementation are cost-effective. ${ }^{97,116,141}$ Rodrigues, Lockwood, Krishnamurthy and Penna also argued that contact screening and SDR-PEP administration raise ethical problems because the disclosure of the index-patients' diagnosis to their contacts is required. ${ }^{138,139}$ However, the LPEP program results in Brazil illustrated that it is possible to distribute SDR-PEP without disclosing the identity of the index case by solely saying: "There is leprosy in your area, and that is why we offer people preventive treatment against leprosy" (Ignotti, unpublished report).

The LPEP program successfully addressed several concerns by demonstrating the feasibility and acceptability of making prevention, in the form of contact screening and SDRPEP administration, part of leprosy control. Embedding the approach in existing leprosy services invigorated the leprosy program. Two additional systematic reviews of quantitative and qualitative data and a meta-analysis in 2017 and 2018 confirmed the effectiveness of SDR as PEP and the general acceptance of the approach. ${ }^{142,143}$

Preventive chemotherapy has become part of leprosy control. This is needed, since the incidence has been more or less stable at around 210,000 in the past 10 years. $^{7}$ Several high-burden countries (eg, India and Indonesia) have embedded SDR-PEP into routine leprosy control nationwide. ${ }^{122,123,144}$ Subsequent to the publication of the latest WHO guidelines that recommend implementing SDR-PEP, many other countries are following suit. ${ }^{52}$ Recent modeling studies at Erasmus MC Rotterdam have estimated that a large-scale roll-out of SDR-PEP may reduce the incidence of leprosy by $50 \%$ in $5-6$ years (Taal et al, under publication).

In a strategy to halt leprosy transmission, a possible leprosy endgame strategy was described comprising contact screening and chemoprophylaxis. ${ }^{110}$ Additionally, the Leprosy Research Initiative (LRI) and the Global Partnership for Zero Leprosy (GPZL) described research priorities related to PEP. ${ }^{145,146,155}$ This included the need to seek more effective regimens, especially for household and blood-related contacts.

Investments in continued (operational) research are needed to discover the most feasible and acceptable approaches to integrate SDR-PEP into leprosy control programs in different contexts. Da Cunha et al recommended that if SDR-PEP was implemented in Brazil, it should start on a small scale, generating new evidence from the Brazilian context. ${ }^{147}$

Several post-exposure chemoprophylactic studies against leprosy are ongoing. The PEP4LEP project in Ethiopia, Tanzania, and Mozambique compares the feasibility of 
a health center-based contact approach to acommunity skin camp-based approach regarding integrated skin screening and SDR-PEP administration (Schoenmakers et al, under publication). In the skin camp group, disclosing the disease status of an (index) leprosy patient is not expected to be necessary.

Other initiatives are working on an improved regimen as chemoprophylaxis for leprosy. An enhanced antibiotic regimen is being tested in the $\mathrm{PEP}++$ project, conducted in India, Brazil, and Indonesia, comprising three standard weight-adjusted single doses of rifampicin plus clarithromycin, given at four weekly intervals. ${ }^{148}$ The effectiveness of $\mathrm{PEP}++$ still has to be established but is promising as a regimen. The expert meeting on defining an enhanced PEP regimen for leprosy discussed other options for improving PEP, including the use of more potent and/or longer-acting antibiotics, such as bedaquiline, rifapentine, oxazolidinone, and nitro-dihydro-imidazo-oxazoles. ${ }^{148}$ These antibiotics were excluded at the time because they were not yet registered in the countries included in the $\mathrm{PEP}++$ study (India, Brazil, Indonesia) or because no experience was gained with these drugs in leprosy treatment. Clarithromycin was chosen after moxifloxacin was deemed unsuitable for prophylactic interventions because of the potential side-effects (publication expected). ${ }^{149}$

In the meantime, WHO has widely pushed the licensing of selected antibiotics because of their potential as second-line treatment in multidrug-resistant TB (MDR-TB), so there is ample opportunity to research their efficacy in preventive chemotherapy for leprosy. The advantages of higher dosages of rifampicin as chemoprophylaxis are currently being investigated by the PEOPLE project. Ortuno-Gutierrez et al have published the protocol of this study which is conducted on the Comoros and on Madagascar. ${ }^{136,146}$ Instead of the $10 \mathrm{mg} / \mathrm{kg}$ rifampicin dose which was used in the COLEP study and the LPEP program, a double dose of $20 \mathrm{mg} / \mathrm{kg}$ is used in the PEOPLE project. The study will use an antiPGL-I test in one of its arms, in which the SDR double dose will be administered only to contacts who test positive for the anti-PGL-I test.

Improved understanding of leprosy transmission routes will facilitate the design of targeted interventions that complement early case detection and prophylaxis. When a fieldfriendly diagnostic (rapid) test becomes available - especially a test indicating who is infected with $M$. leprae, incubating and transmitting leprosy before signs or symptoms occur-targeted chemoprophylaxis for leprosy in certain groups, such as household members, would be an option. ${ }^{146,150,151}$ Such a test would allow a more tailored approach at the individual level (personalized medicine), which may increase efficacy of PEP for blood-related household and other high-risk contacts. ${ }^{146}$ The GPZL PEP research agenda includes the question: "Which type of PEP intervention fits best with which epidemiological setting?", which requires more operational research. ${ }^{146}$ In very high-endemic hotspot or cluster areas, a total population screening and SDR-PEP administration may be the best approach. ${ }^{101,116}$ However, when screening entire populations is neither feasible nor affordable, focal mass drug administration (fMDA) may be a reasonable alternative. ${ }^{152}$ The fMDA approach would target the population who are not included in a contact-based PEP approach and would be implemented alongside contact-based PEP. The effectiveness and feasibility of such an approach first needs to be tested in a randomized controlled trial. Variations on screening, such as self-screening or self-referred screening only, need to be evaluated. An additional benefit of fMDA would be that it is especially suitable in areas where stigma prevents patients from disclosing their disease status.

Questions concerning disease concealment for index patients, optimal contact and community education (eg, regarding the fact that chemoprophylaxis is not $100 \%$ effective), and the quality of leprosy screening by health workers need to be addressed in future research projects. ${ }^{146}$

Another important topic is antibiotic resistance. Even though the risk of inducing rifampicin resistance in M. tuberculosis is considered negligible, regular sampling and molecular monitoring for mutations associated with rifampicin resistance in $M$. tuberculosis and in $M$. leprae as recommended by WHO are encouraged in areas with a high rate of primary MDR-TB and among recipients of SDR-PEP who develop leprosy. ${ }^{113,146,153}$

Additionally, loose rifampicin that can be used as SDRPEP for leprosy prevention is urgently needed and should be made available on a global scale to facilitate further SDRPEP implementation. Rifampicin is relatively cheap, and its cost-effectiveness was assessed to be good in the COLEP study, and also in India, based on the SIMCOLEP model and the LPEP program. ${ }^{97,116}$ Through the Stop TB Partnership/Global Drug Facility, 100 dosages of $150 \mathrm{mg}$ rifampicin cost US\$ 6.33, and 100 dosages of $300 \mathrm{mg}$ cost around US\$ $10 .{ }^{154}$ But SDR-PEP still requires an initial investment from national leprosy control programs in often resource-poor settings, especially when SDR-PEP administration is - as recommended - combined with screening and active case-finding activities. ${ }^{52,150}$ Free provision, similar to 
MDT, would be beneficial on a global scale. Additionally, registration of the new indication (post-exposure prophylaxis in leprosy) of loose rifampicin in more national registers of authorized medicines should be initiated by ministries of health in leprosy endemic countries. Furthermore, logistical structures and (national) registration systems, for contacts of leprosy patients and PEP pharmaceutical stock, sould be set up. $^{121}$

As mentioned, Taal et al have estimated the number of persons that need to be treated with SDR-PEP using the SIMCOLEP model (Taal et al, under publication). The model not only predicts the number of people who need to be treated with SDR-PEP to achieve a 50\% reduction in new leprosy case detection at a global level, but it also estimates how many people need chemoprophylaxis with rifampicin for a 90\% leprosy case reduction. Predictions per country will also be published. This model and the other available evidence make clear that the implementation of evidence-based preventive interventions against leprosy, like SDR-PEP, in national programs needs to be encouraged to facilitate a large-scale roll-out of PEP. This will help prevent those at risk from developing leprosy and will be a vital step to stop further transmission of this ancient disease. Last, but not least, it will prevent more people from having to live with leprosy and its physical, psychological, and socioeconomic consequences.

\section{Disclosure}

NLR was a co-sponsor and partner in the Indonesia PEP trial (2000-2005; multiple funders), the COLEP trial (2002-2008; multiple funders) and the LPEP program (implementation period 2015-2018; funded by Novartis Foundation). Currently, NLR works on several projects including PEP++ (funding from the Dutch Postcode Lottery) and PEP4LEP (funding from the second European and Developing Countries Clinical Trials Partnership program [EDCTP2] supported by the European Union under grant number RIA2017NIM1839-PEP4LEP, and from the Leprosy Research Initiative [LRI] under grant number 707.19.58). For this review, all authors report no conflicts of interest.

\section{References}

1. Lastória JC, de Abreu MAMM. Leprosy: review of the epidemiological, clinical, and etiopathogenic aspects - part 1. An Bras Dermatol. 2014;89(2):205-218. doi:10.1590/abd1806-4841.20142450
2. Smith CS, Noordeen SK, Richardus JH, et al. A strategy to halt leprosy transmission. Lancet Infect Dis. 2014;14(2):96-98. doi:10.1016/S1473-3099(13)70365-7

3. World Health Organization. Leprosy - key facts. [Published September 10, 2019]. Available from: https://www.who.int/newsroom/fact-sheets/detail/leprosy. Accessed February 8, 2020.

4. Manderson L. Neglected diseases of poverty. Med Anthropol Cross Cult Stud Heal Illn. 2012;31(4):283-286. doi:10.1080/ 01459740.2012.693441

5. Bhat RM, Prakash C. Leprosy: an overview of pathophysiology. Interdiscip Perspect Infect Dis. 2012;2012. Doi:10.1155/2012/181089

6. Leonardo L, Hernandez L, Magturo TC, et al. Current status of neglected tropical diseases (NTDs) in the Philippines. Acta Trop. 2020;203:105284. doi:10.1016/j.actatropica.2019.105284

7. World Health Organization. Global leprosy update, 2018: moving towards a leprosy-free world. Wkly Epidemiol Rec. 2019;94(35/ 36):389-411.

8. World Health Organization. Leprosy: the disease. Published 2016. Available from: https://www.who.int/lep/leprosy/en/. Accessed February 8, 2020.

9. Storrs EE, Walsh GP, Burchfield HP, Binford CH. Leprosy in the armadillo: new model for biomedical research. Science. 1974;183 (4127):851-852. doi:10.1126/science.183.4127.851

10. Colston MJ. Application of the thymectomized-irradiated mouse to the detection of persisting Mycobacterium leprae. Int J Lepr. 1988;55(4):859-863.

11. Rinaldi A. The global campaign to eliminate leprosy. PLoS Med. 2005;2(12):1222-1225. doi:10.1371/journal.pmed.0020341

12. Moet FJ, Pahan D, Schuring RP, Oskam L, Richardus JH. Physical distance, genetic relationship, age, and leprosy classification are independent risk factors for leprosy in contacts of patients with leprosy. J Infect Dis. 2006;193(3):346-353. doi:10.1086/499278

13. Van Beers SM, Hatta M, Klatser PR. Patient contact is the major determinant in incident leprosy: implications for future control. Int J Lepr Other Mycobact Dis. 1999;67(2):119-128.

14. Hoeven TA, Fischer EAJ, Pahan D, Richardus JH. Social distance and spatial distance are not the same, observations on the use of GIS in leprosy epidemiology. Epidemiol Infect. 2008;136 (12):1624-1627. doi:10.1017/S0950268808000381

15. World Health Organization Souht-East Asia. Leprosy: fact sheet.

16. Monteiro YN. Prophylaxis and exclusion: compulsory isolation of Hansen's disease patients in São Paulo. Hist Cienc Saude Manguinhos. 2003;10(Suppl 1):95-121. doi:10.1590/S010459702003000400005

17. Muir E. Editorials - first congress of the international leprosy association. Fourth international leprosy conference. Int J Lepr. 1937;5(1):87-91.

18. Noordeen SK. Elimination of leprosy as a public health problem: progress and prospects. Bull World Health Organ. 1995;73(1):1-6.

19. Meima A, Cairns W, Smith S, et al. The future incidence of leprosy: a scenario analysis. Bull World Health Organ. 2004;82:373-380.

20. Vijayakumaran P, Krishnamurthy P, Rao P, et al. Chemoprophylaxis against leprosy: expectations and methodology of a trial. Lepr Rev. 2000;71:S37-S41. doi:10.5935/03057518.20000065

21. Smith WC, van Brakel W, Gillis T, Saunderson P, Richardus JH. The missing millions: a threat to the elimination of leprosy. PLoS Negl Trop Dis. 2015;9(4):e0003658. doi:10.1371/journal.pntd.0003658

22. World Health Organization. Global Leprosy Strategy 2016-2020: Accelerating Towards a Leprosy-Free World. World Health Organization; 2016.

23. Richardus JH, Pahan D, Bangladesh L, et al. Leprosy Control International Textbook of Leprosy. Scollard DM, Gillis TP, editors. Available from: https://internationaltextbookofleprosy.org/. Accessed February 8, 2020 
24. Barth-Jaeggi T, Steinmann P, Mieras L, et al. Leprosy post-exposure prophylaxis (LPEP) programme: study protocol for evaluating the feasibility and impact on case detection rates of contact tracing and single dose rifampicin. BMJ Open. 2016;6 (11):e013633. doi:10.1136/bmjopen-2016-013633

25. Haneem F, Ali R, Kama N, Basri S Descriptive analysis and text analysis in systematic literature review: a review of master data management. In: International Conference on Research and Innovation in Information Systems, ICRIIS; 2017. IEEE Computer Society. doi:10.1109/ICRIIS.2017.8002473

26. Infolep. International knowledge center for information resources on leprosy | infolep. Available from: https://www.leprosyinformation.org/. Accessed February 8, 2020.

27. Moher D, Liberati A, Tetzlaff J, et al. Preferred reporting items for systematic reviews and meta-analyses: the PRISMA statement. PLoS Med. 2009;6(7):e1000097. doi:10.1371/journal.pmed.1000097

28. Luca S, Mihaescu T. History of BCG vaccine. Maedica. 2013;8 (1):53-58.

29. World Health Organization. BCG vaccine: WHO position paper, February 2018 - recommendations. Vaccine. 2018;36 (24):3408-3410. Doi:10.1016/j.vaccine.2018.03.009

30. Seki M, Honda I, Fujita I, Yano I, Yamamoto S, Koyama A. Whole genome sequence analysis of Mycobacterium bovis bacillus Calmette-Guérin (BCG) Tokyo 172: a comparative study of BCG vaccine substrains. Vaccine. 2009;27(11):1710-1716. doi:10.1016/j.vaccine.2009.01.034

31. Cernuschi T, Malvolti S, Nickels E, Friede M. Bacillus CalmetteGuérin (BCG) vaccine: a global assessment of demand and supply balance. Vaccine. 2018;36(4):498-506. doi:10.1016/j. vaccine.2017.12.010

32. Kinnear Brown JA, Stone MM. Vaccination against leprosy. $\mathrm{Br}$ Med J. 1966;1(5478). http://www.ncbi.nlm.nih.gov/pmc/articles/ PMC1845248/pdf/brmedj02531-0013.pdf.

33. Fernandez JMM. Leprosy and tuberculosis: antagonistic diseases. A M a Arch Dermatology. 1957;75(1):101-106. doi:10.1001/ archderm.1957.01550130103011

34. Richardus R, van Hooij A, van den Eeden SJF, et al. BCG and adverse events in the context of leprosy. Front Immunol. 2018;9:629. doi:10.3389/fimmu.2018.00629

35. Setia MS, Steinmaus C, Ho CS, Rutherford GW. The role of BCG in prevention of leprosy: a meta-analysis. Lancet Infect Dis. 2006;6(3):162-170. doi:10.1016/S1473-3099(06)70412-1

36. Zodpey SP, Bansod BS, Shrikhande SN, Maldhure BR, Kulkarni SW. Protective effect of Bacillus Calmette Guerin (BCG) against leprosy: a population-based case-control study in Nagpur, India. Lepr Rev. 1999;70(3):287-294. doi:10.5935/03057518.19990032

37. Merle C, Cunha S, Rodrigues L. BCG vaccination and leprosy protection: review of current evidence and status of BCG in leprosy control. Expert Rev Vaccines. 2010;9(2):209-222. doi:10.1586/erv.09.161

38. Zodpey SP, Ambadekar NN, Thakur A. Effectiveness of Bacillus Calmette Guerin (BCG) vaccination in the prevention of leprosy: a population-based case-control study in Yavatmal District, India. Public Health. 2005;119(3):209-216. doi:10.1016/j.puhe.2004.04. 007

39. Rodrigues LC, LRS K-P, Frietas MVC, Barreto ML. Long lasting BCG protection against leprosy. Vaccine. 2007;25(39-40):6842-6844. doi:10.1016/j.vaccine.2007.07.032

40. Smith WC, Saunderson P. Leprosy. BMJ Clin Evid. 2011;2010:0915.

41. Yanagisawa K. The effect of BCG vaccination upon the occurrence of leprosy in nursery children. Int J Lepr. 1959;26 (4):325-327.

42. Price JE. BCG vaccination in leprosy. Int J Lepr. 1982;50 (2):205-212
43. Lwin K, Sundaresan T, Gyi MM, et al. BCG vaccination of children against leprosy: fourteen-year findings of the trial in Burma. Bull World Health Organ. 1985;63(6):1069-1078.

44. Richardus RA, Alam K, Pahan D, Feenstra SG, Geluk A, Richardus JH. The combined effect of chemoprophylaxis with single dose rifampicin and immunoprophylaxis with BCG to prevent leprosy in contacts of newly diagnosed leprosy cases: a cluster randomized controlled trial (MALTALEP study). BMC Infect Dis. 2013;13(1). doi:10.1186/1471-2334-13-456

45. Goulart IMBMB, Goulart LRR. Leprosy: diagnostic and control challenges for a worldwide disease. Arch Dermatol Res. 2008;300 (6):269-290. doi:10.1007/s00403-008-0857-y

46. de Matos HJ, Duppre N, Alvim MF, MachadoVieira LM, Sarno EN, Struchiner CJ. Leprosy epidemiology in a cohort of household contacts in Rio de Janeiro (1987-1991). Cadernos De Saúde Pública. 1999;15(3):533-542. doi:10.1590/S0102-311X19 99000300010

47. Stanley SJ, Howland C, Stone MM, Sutherland I. BCG vaccination of children against leprosy in Uganda: final results. $J$ Hyg. 1981;87(2):233-248. doi:10.1017/S002217240006945X

48. Düppre NC, Camacho LAB, da Cunha SS, et al. Effectiveness of BCG vaccination among leprosy contacts: a cohort study. Trans $R$ Soc Trop Med Hyg. 2008;102(7):631-638. doi:10.1016/j. trstmh.2008.04.015

49. Duthie M, Balagon M. Combination chemoprophylaxis and immunoprophylaxis in reducing the incidence of leprosy. Risk Manag Healthc Policy. 2016;9:43-53. doi:10.2147/RMHP.S76058

50. Sharma P, Mukherjee R, Talwar GP, et al. Immunoprophylactic effects of the anti-leprosy Mw vaccine in household contacts of leprosy patients: clinical field trials with a follow up of 8-10 years. Lepr Rev. 2005;76(2):127-143.

51. Gupte MD, Vallishayee RS, Anantharaman DS, et al. Comparative leprosy vaccine trial in south India. Indian J Lepr. 1998;70(4):369-388.

52. World Health Organization. Guidelines for the diagnosis, treatment and prevention of leprosy. New Delhi: World Health Organization; 2018. Available from: http://apps.who.int/iris/han dle/10665/274127.

53. Convit J, Ulrich M, Aranzazu N. Vaccination in leprosy. Observations and interpretations. Int J Lepr. 1980;48(1):62-65.

54. Karonga Prevention Trial Group. Randomised controlled trial of single BCG, repeated BCG, or combined BCG and killed Mycobacterium leprae vaccine for prevention of leprosy and tuberculosis in Malawi. Karonga Prevention Trial Group. Lancet. 1996;348(9019):17-24. doi:10.1016/S0140-6736(96) 02166-6

55. Truoc L, Ly H, Thuy N, Trach D, Stanford C, Stanford J. Vaccination against leprosy at Ben San Leprosy Centre, Ho Chi Minh City, Vietnam. Vaccine. 2001;19(25-26):3451-3458. doi:10.1016/s0264-410x(01)00052-4

56. Coppola M, van den Eeden SJF, Robbins N, et al. Vaccines for leprosy and tuberculosis: opportunities for shared research, development, and application. Front Immunol. 2018;9:308. doi:10.33 89/fimmu.2018.00308

57. Duthie M, Pena M, Ebenezer G, et al. LepVax, a defined subunit vaccine that provides effective pre-exposure and post-exposure prophylaxis of $\mathrm{M}$. leprae infection. $N p j$ Vaccines. 2018;3(1):1-9.

58. Kamal R, Pathak V, Kumari A, Natrajan M, Katoch K, Kar H. Addition of Mycobacterium indicus pranii (MIP) vaccine as an immunotherapeutic with standard chemotherapy in borderline leprosy: a double blind study to assess clinical improvement (a preliminary report). $\mathrm{Br} J$ Dermatol. 2017;176:1388-1389.

59. Katoch K. Review of global leprosy vaccine development. Indian J Lepr. 2012;84(1):70-71. 
60. Duthie MS, Casper C, Reed SG. Second coming: the re-emergence and modernization of immunotherapy by vaccines as a component of leprosy control. Future Microbiol. 2018;13 (13):1449-1451. doi:10.2217/fmb-2018-0186

61. Talwar GP, Singh P, Atrey N, Gupta JC. Making of a highly useful multipurpose vaccine. Res Artic J Transl Sci J Transl Sci. 2016;1(3):69-73. doi:10.15761/JTS.1000117

62. Talwar GP, Ahmed N, Saini V. The use of the name Mycobacterium w for the leprosy immunotherapeutic bacillus creates confusion with $M$. tuberculosis-W (Beijing strain): a suggestion. Infect Genet Evol. 2008;8(1):100-101. doi:10.1016/j. meegid.2007.07.009

63. Kamal M, Natrajan R, Katoch K, Arora M. Clinical and histopathological evaluation of the effect of addition of immunotherapy with $\mathrm{Mw}$ vaccine to standard chemotherapy in borderline leprosy. Indian J Lepr. 2012;84(4):287-306.

64. Kamal R, Natrajan M, Dayal R. Addition of immunotherapy to chemotherapy in pediatric borderline leprosy: a clinical evaluation. Int $J$ Contemp Pediatr. 2016:1439-1444. Doi:10.18203/2349-3291.ijcp20163695

65. International Federation of Anti-Leprosy Associations. The leprosy vaccine. Published 2019. Available from: https://www. ilepfederation.org/ilep-update-november-2019/the-leprosyvaccine/. Accessed February 11, 2020.

66. Dharmendra M. Prophylactic value of dapsone in leprosy. J Am Med Assoc. 1966;195(9):787. doi:10.1001/jama.1966.03100090121035

67. Lew J, Kim YS. Chemoprophylaxis of leprosy contacts with D.D. S. Yonsei Med J. 1966;7:47-51. doi:10.3349/ymj.1966.7.1.47

68. Wardekar R. DDs prophylaxis against leprosy. Lepr India. 1967;39(4):155-159.

69. Otsyula Y, Ibworo C, Chum HJ, Four years' experience with dapsone as prophylaxis against leprosy. Lepr Rev. 1971;42 (2):98-100. doi:10.5935/0305-7518.19710012

70. Noordeen SK, Neelan PN. Chemoprophylaxis among contacts of non-lepromatous leprosy. Lepr India. 1976;48(4 Suppl):635-642.

71. Noordeen SK. Long-term effects of chemoprophylaxis among contacts of lepromatous cases. Results of a 8.5 years follow-up. Lepr India. 1977;49(4):504-509.

72. Koticha K, Nair P. Antileprosy measures in Bombay, India: an analysis of 10 years' work. Bull World Health Organ. 1976;54(1):67-77.

73. Dayal R, Bharadwaj VP. Prevention and early detection of leprosy in children. J Trop Pediatr. 1995;41(3):132-138. doi:10.1093/ tropej/41.3.132

74. Filice GA, Fraser DW. Management of household contacts of leprosy patients. Ann Intern Med. 1978;88(4):538-542. doi:10.7326/0003-4819-88-4-538

75. Sloan NR, Jano B, Worth RM, Fasal P, Shepard CC. Repository acedapsone in leprosy chemoprophylaxis and treatment. Lancet 1971;298(7723):525-526. doi:10.1016/S0140-6736(71)90442-9

76. Russell DA, Worth RM, Scott GC, et al. Experience with acedapsone (DADDS) in the therapeutic trial in New Guinea and the chemoprophylactic trial in Micronesia. Int $J$ Lepr. 1976;44 (1-2):170-176.

77. Neelan PN, Noordeen SK, Sivaprasad N. Chemoprophylaxis against leprosy with acedapsone. Indian J Med Res. 1983;78 (3):307-313.

78. Neelan PN, Sirumban P, Sivaprasad N. Limited duration acedapsone prophylaxis in leprosy. Indian J Lepr. 1986;58(2):251-256.

79. Smith CM, Smith WC. Chemoprophylaxis is effective in the prevention of leprosy in endemic countries: a systematic review and meta-analysis. MILEP2 study group. Mucosal immunology of leprosy. J Infect. 2000;41(2):137-142. doi:10.1053/jinf.2000.0698

80. Blanc LJ, Brennan PJ, Cho SN, et al. Workshop on the prevention of leprosy, Pohnpei, Federated States of Micronesia, 25-27 May 1999. In: International Journal of Leprosy and Other Mycobacterial Diseases. Vol 67. 1999.
81. Cartel JL, Chanteau S, Boutin JP, et al. Implementation of chemoprophylaxis of leprosy in the Southern Marquesas with a single dose of $25 \mathrm{mg}$ per $\mathrm{kg}$ rifampin. Int J Lepr Other Mycobact Dis. 1989;57(4):810-816.

82. Cartel JL, Chanteau S, Moulia-Pelat JP, et al. Chemoprophylaxis of leprosy with a single dose of $25 \mathrm{mg}$ per $\mathrm{kg}$ rifampin in the Southern Marquesas; Results after four years. Int J Lepr. 1992;60(3):416-420.

83. Cho SN, Walsh GP, Brennan PJ. Monitoring the effects of preventive therapy in the Federated States of Micronesia. Int J Lepr Other Mycobact Dis. 1999;67(4 Supp1):S19-22.

84. Diletto C. Elimination of leprosy in the federated states of micronesia by intensive case finding, treatment with WHO/MDT and administration of chemoprophylaxis. Int J Lepr Other Mycobact Dis. 1999;67(4 Suppl):S10-3.

85. Takashima J. Implementation of chemoprophylaxis in Chuuk State, Federated States of Micronesia. Int J Lepr Other Mycobact Dis. 1999;67(4 Suppl):S16-8

86. Iehsi-Keller E. Implementation of chemoprophylaxis in Pohnpei State, Federated States of Micronesia. Int J Lepr Other Mycobact Dis. 1999;67(4 Suppl):S14-5.

87. Daulako EC. Population screening and mass chemoprophylaxis in Kiribati. Int J Lepr Other Mycobact Dis. 1999;67(4 Suppl):S23-5.

88. Tin K. Population screening and chemoprophylaxis for household contacts of leprosy patients in the Republic of the Marshall Islands. Int J Lepr Other Mycobact Dis. 1999;67(4 Suppl):S26-9.

89. Ji B, Grosset JH. Drugs and regimens for preventive therapy against tuberculosis, disseminated Mycobacterium avium complex infection and leprosy. Int J Lepr Other Mycobact Dis. 1999;67(4):s45.

90. Noordeen SK. The future of leprosy elimination. Int J Lepr Other Mycobact Dis. 1999;67(4):s56.

91. Lechat MF. Rationale for the preventive treatment of leprosy. Int J Lepr Other Mycobact Dis. 1999;67(4):s63.

92. Nguyen LN, Cartel JL, Grosset JH, Nguyen LN, Cartel JL, Grosset JH. Chemoprophylaxis of leprosy in the Southern Marquesas with a single $25 \mathrm{mg} / \mathrm{kg}$ dose of rifampicin. Results after 10 years. Lepr Rev. 2000;71:S33-S36. doi:10.5935/03057518.20000064

93. Diletto C, Blanc L, Levy L. Leprosy chemoprophylaxis in Micronesia. Lepr Rev. 2000;71:S21-3;discussion S24-5. doi:10.5935/0305-7518.20000061

94. Atrio Mouriño CN, Rodríguez Del Valle KM, Gutiérrez KYS, Ávila MM, Rodríguez ESS. Eficacia de la rifampicina como profiláctico en contactos de primer orden de lepra. Rev Leprol. 2019;32(2):87-104.

95. Moet FJ, Oskam L, Faber R, Pahan D, Richardus JH. A study on transmission and a trial of chemoprophylaxis in contacts of leprosy patients: design, methodology and recruitment findings of COLEP. Lepr Rev. 2004;75(4):376-388.

96. Moet FJ, Pahan D, Oskam L, Richardus JH. Effectiveness of single dose rifampicin in preventing leprosy in close contacts of patients with newly diagnosed leprosy: cluster randomised controlled trial. BMJ. 2008;336(7647):761-764. doi:10.1136/bmj.39 500.885752.BE

97. Idema WJ, Majer IM, Pahan D, Oskam L, Polinder S, Richardus JH. Cost-effectiveness of a chemoprophylactic intervention with single dose rifampicin in contacts of new leprosy patients. PLoS Negl Trop Dis. 2010;4(11):e874. doi:10.1371/journal.pntd.0000874

98. Schuring RP, Richardus JH, Pahan D, Oskam L. Protective effect of the combination BCG vaccination and rifampicin prophylaxis in leprosy prevention. Vaccine. 2009;27(50):7125-7128. doi:10.1016/j.vaccine.2009.09.054

99. Feenstra SG, Nahar Q, Pahan D, Oskam L, Richardus JH. Acceptability of chemoprophylaxis for household contacts of leprosy patients in Bangladesh: a qualitative study. Lepr Rev. 2011;82(2):178-187. 
100. Oskam L, Bakker MI. Report of the workshop on the use of chemoprophylaxis in the control of leprosy held in Amsterdam, the Netherlands on 14 December 2006. Lepr Rev. 2007;78(2):173-185.

101. Bakker MI, Hatta M, Kwenang A, et al. Prevention of leprosy using rifampicin as chemoprophylaxis. Am J Trop Med Hyg. 2005;72(4):443-448. doi:10.4269/ajtmh.2005.72.443

102. Reveiz L, Buendía JA, Téllez D. Chemoprophylaxis in contacts of patients with leprosy: systematic review and meta-analysis. Rev Panam Salud Pública. 2009;26:341-349. doi:10.1590/ S1020-49892009001000009

103. World Health Organization South-East Asia. Monitoring grade-2 disability rate and applicability of chemoprophylaxis in leprosy control: report of the information consultation, London, United Kingdom, 12-13 November 2009. New Delhi: WHO Regional Office for South-East Asia; 2010.

104. van Brakel W, Cross H, Declercq E, et al. Review of leprosy research evidence (2002-2009) and implications for current policy and practice. Lepr Rev. 2010;81(3):228-275.

105. Idrissi A, Khoudri I, Sekkat A, Ismaili N, Latifi A, Hali F. Guide De La Lutte Antilepreuse. 2014.

106. Khoudri I, Elyoussfi Z, Mourchid Y, et al. Trend analysis of leprosy in Morocco between 2000 and 2017: evidence on the single dose rifampicin chemoprophylaxis. PLoS Negl Trop Dis. 2018;12(12):e0006910. doi:10.1371/journal.pntd.0006910

107. Fischer EAJ, De Vlas SJ, Habbema JDF, Richardus JH. The long term effect of current and new interventions on the new case detection of leprosy: a modeling study. PLoS Negl Trop Dis. 2011;5(9):e1330. doi:10.1371/journal.pntd.0001330

108. de Matos HJ, Blok DJ, de Vlas SJ, et al. Leprosy new case detection trends and the future effect of preventive interventions in Pará State, Brazil: a modelling study. PLoS Negl Trop Dis. 2016;10(3):1-10. doi:10.1371/journal.pntd.0004507

109. Gilkison C, Chambers S, Blok DJ, et al. Predicting the impact of household contact and mass chemoprophylaxis on future new leprosy cases in South Tarawa, Kiribati: a modelling study. PLoS Negl Trop Dis. 2019;13(9):e0007646. doi:10.1371/journal. pntd.0007646

110. Smith WCS, Aerts A. Role of contact tracing and prevention strategies in the interruption of leprosy transmission. Lepr Rev. 2014;85(1):2-17.

111. Richardus JH, Meima A, Van Marrewijk CJ, Croft RP, Smith TC. Close contacts with leprosy in newly diagnosed leprosy patients in a high and low endemic area: comparison between Bangladesh and Thailand. Int J Lepr Other Mycobact Dis. 2005;73(4):249-257.

112. Richardus JH. Chemoprophylaxis: sufficient evidence for starting implementation pilots. Lepr Rev. 2015;86(1):128-129.

113. Mieras L, Anthony R, van Brakel W, et al. Negligible risk of inducing resistance in Mycobacterium tuberculosis with single-dose rifampicin as post-exposure prophylaxis for leprosy. Infect Dis Poverty. 2016;5(1):46. doi:10.1186/s40249-016-0140-y

114. Steinmann P, Cavaliero A, Aerts A, et al. The leprosy post-exposure prophylaxis (LPEP) programme: update and interim analysis. Lepr Rev. 2018;89(2):102-116.

115. Richardus JH, Cavaliero A, Steinmann P. Abstracts of oral presentations - 175 feasibility and impact of leprosy post-exposure prophylaxis: evidence from LPEP, a multi-country, 5-year implementation research program. Trans $R$ Soc Trop Med Hyg. 2019;113:S71. doi:10.1093/trstmh/trz094

116. Tiwari A, Blok DJ, Arif M, Richardus JH. Leprosy post-exposure prophylaxis in the Indian health system: A cost-effectiveness analysis. PLoS Negl Trop Dis. 2020 Aug 4;14(8):e0008521. doi: 10.1371/ journal.pntd.0008521. PMID: 32750059; PMCID: PMC7428216.

117. Cavaliero A, Greter H, Fürst T, et al. An innovative approach to screening and chemoprophylaxis among contacts of leprosy patients in low endemic settings: experiences from Cambodia. PLoS Negl Trop Dis. 2018;13(3). doi:10.1371/journal.pntd.0007039
118. Peters R, Mieras L, Subedi M, et al. A single dose of rifampicin to prevent leprosy: qualitative analysis of perceptions of persons affected, contacts, community members and health professionals towards chemoprophylaxis and the impact on their attitudes in India, Nepal and Indonesia. Lepr Rev. 2018;89(4):335-352.

119. Apte H, Chitale M, Das S, Manglani PR, Mieras LF. Acceptability of contact screening and single dose rifampicin as chemoprophylaxis for leprosy in Dadra and Nagar Haveli, India. Lepr Rev. 2019;90(1):31-45.

120. Barth-Jaeggi T, Cavaliero A, Aerts A. Leprosy post-exposure prophylaxis with single-dose rifampicin: toolkit for implementation. Lepr Rev. 2019;90(4):356-363.

121. Richardus JH, Kasang C, Mieras L, et al. Minimal essential data to document contact tracing and single dose rifampicin (SDR) for leprosy control in routine settings: a practical guide. Lepr Rev. 2018;89(1):2-12.

122. Gillini L, Cooreman E, Wood T, Pemmaraju VR, Saunderson P. Global practices in regard to implementation of preventive measures for leprosy. PLoS Negl Trop Dis. 2017;11(5):e0005399.

123. Menteri Kesehatan Republik Indonesia. Peraturan Menteri Kesehatan Republik Indonesia, Nomor 11 Tahun 2019 Tentang Penanggulangan Kusta. 2019.

124. Addiss DG. Evidence, opportunity, ethics, and the allure of zero leprosy. Lepr Rev. 2018;89(2):90-101.

125. Richardus JH, Geluk A. Reply to: single-dose rifampicin and BCG to prevent leprosy. Int J Infect Dis. 2020;92:271-272. doi:10.1016/j.ijid.2020.01.052

126. Richardus R, Alam K, Kundu K, et al. Effectiveness of single-dose rifampicin after BCG vaccination to prevent leprosy in close contacts of patients with newly diagnosed leprosy: a cluster randomized controlled trial. Int $J$ Infect Dis. 2019;88:65-72. doi:10.1016/j.ijid.2019.08.035

127. Richardus RA, Butlin CR, Alam K, Kundu K, Geluk A, Richardus JH. Clinical manifestations of leprosy after BCG vaccination: an observational study in Bangladesh. Vaccine. 2015;33 (13):1562-1567. doi:10.1016/j.vaccine.2015.02.017

128. Gomes RR, Antunes DE, Dos Santos DF, Sabino EFP, Oliveira DB, Goulart IMB. BCG vaccine and leprosy household contacts: protective effect and probability to becoming sick during follow-up. Vaccine. 2019;37(43):6510-6517. doi:10.1016/j.vaccine.2019.08.067

129. Gormus BJ, Meyers WM. Under-explored experimental topics related to integral mycobacterial vaccines for leprosy. Expert Rev Vaccines. 2003;2(6):791-804. doi:10.1586/14760584.2.6.791

130. Goulart IMB, Bernardes Souza DO, Marques CR, Pimenta VL, Gonçalves MA, Goulart LR. Risk and protective factors for leprosy development determined by epidemiological surveillance of household contacts. Clin Vaccine Immunol. 2008;15(1):101105. doi: 10.1128/CVI.00372-07

131. Minísterio da Saúde Brasil. Portaria $\mathrm{N}^{\mathrm{o}} 3.125$, de 7 de Outubro de 2010. Diretrizes Para Vigilância, Atenção e Controle Da Hansenías E. 2010.

132. Floyd S, Ponnighaus JM, Bliss L, et al. BCG scars in northern Malawi: sensitivity and repeatability of scar reading, and factors affecting scar size. Int J Tuberc Lung Dis. 2000;4(12):1133-1142.

133. Goulart IMB, Bernardes Souza DO, Marques CR, Pimenta VL, Gonçalves MA, Goulart LR. Risk and protective factors for leprosy development determined by epidemiological surveillance of household contacts. Clin Vaccine Immunol. 2008;15 (1):101-105. doi:10.1128/CVI.00372-07

134. Cunha S, Alexander N, Barreto M, et al. BCG revaccination does not protect against leprosy in the Brazilian Amazon: a cluster randomised trial. PLoS Negl Trop Dis. 2008;2(2):e167.

135. Duthie M, Coler R, Laurance J, et al. Protection against M. leprae infection by the ID83/GLA-SE and ID93/GLA-SE vaccines developed for tuberculosis. Infect Immun. 2014;82 (9):3979-3985. 
136. Ortuno-Gutierrez N, Younoussa A, Randrianantoandro A, et al. Protocol, rationale and design of PEOPLE (Post ExpOsure Prophylaxis for LEprosy in the Comoros and Madagascar): a cluster randomized trial on effectiveness of different modalities of implementation of post-exposure prophylaxis of leprosy contacts. BMC Infect Dis. 2019;19(1):1-7. doi:10.1186/s12879-019-4649-0

137. Rahman M, Leprosy chemoprophylaxis: what's the need? BMJ. 2008;336(7649):847-848. doi:10.1136/bmj.39549.445521.3A

138. Rodrigues LC, Lockwood DN. Leprosy now: epidemiology, progress, challenges, and research gaps. Lancet Infect Dis. 2011;11 (6):464-470. doi:10.1016/S1473-3099(11)70006-8

139. Lockwood D, Krishnamurthy PV, Penna G. Reply to the role of contact tracing and prevention strategies in the interruption of leprosy transmission - chemoprophylaxis: a call for more research. Lepr Rev. 2015;86(1):124-125.

140. Lockwood D, Krishnamurthy P, Kumar B, Penna G. Single-dose rifampicin chemoprophylaxis protects those who need it least and is not a cost-effective intervention. PLoS Negl Trop Dis. 2018;12 (6): $\mathrm{e} 0006403$.

141. Ezenduka C, Post E, John S, Suraj A, Namadi A, Onwujekwe O. Cost-effectiveness analysis of three leprosy case detection methods in northern Nigeria. PLoS Negl Trop Dis. 2012;6(9):e1818. doi:10.1371/journal.pntd.0001818

142. Ferreira SMB, Yonekura T, Ignotti E, de Oliveira LB, Takahashi J, Soares CB. Effectiveness of rifampicin chemoprophylaxis in preventing leprosy in patient contacts. JBI Database Syst Rev Implement Rep. 2017;15(10):2555-2584. doi:10.11124/ JBISRIR-2016-003301

143. Ferreira APM, Fan W, Agarwal A, et al. Chemoprophylaxis for contacts of leprosy patients: a systematic review and meta-analysis. Indian J Lepr. 2018;90(3):217-234.

144. National Health Portal of India TM of H and FWG of I. Leprosy. Prevention. Available from: https://www.nhp.gov.in/disease/skin/ leprosy.Published February 26, 2020. Accessed June 15, 2020.

145. Khazai Z, van Brakel W, Essink D, et al. Reviewing research priorities of the leprosy research initiative (LRI): a stakeholder's consultation. Lepr Rev. 2019;90(1):3-30.
146. Kasang C, Steinmann P. Global partnership for zero leprosy research agenda working group subgroup on post-exposure prophylaxis. Glob Partnersh Zero Lepr. 2019;1-9.

147. Da Cunha SS, Bierrenbach AL, Barreto VHL. Chemoprophylaxis to control leprosy and the perspective of its implementation in Brazil: a primer for non-epidemiologists [Quimioprofilaxia para prevenção de hanseníase e sua implantação no Brasil: uma explicação introdutória para não epidemiologistas]. Rev Inst Med Trop Sao Paulo. 2015;57(6):481-487. doi:10.1590/S003646652015000600004

148. Mieras LF, Taal AT, van Brakel WH, et al. An enhanced regimen as post-exposure chemoprophylaxis for leprosy: $\mathrm{PEP}++$. $B M C$ Infect Dis. 2018;18(1):506. doi:10.1186/s12879-018-3402-4

149. European Medicines Agency. Quinolone- and fluoroquinolonecontaining medicinal products.

150. Palit A, Kar H. Prevention of transmission of leprosy: the current scenario. Indian J Dermatol Venereol Leprol. 2020;86:115. doi:10.4103/ijdvl.IJDVL_326_19

151. Naafs B. World Leprosy Day 2018: How forward respecting the past? Indian J Med Res. 2018 Jan;147(1):1-3. doi:10.4103/ijmr. IJMR_109_18

152. Fenenga C. Workshop explores how to scale up PEP and advance research | Global Partnership for Zero Leprosy. Global Partnership for Zero Leprosy.

153. World Health Organization. A guide for surveillance of antimicrobial resistance in leprosy: 2017 update. WHO; August 2017. Available from: https://www.who.int/lep/resources/ 9789290226192/en/. Accessed April 6, 2020.

154. Stop TB Partnership. January 2020 medicines catalog - global drug facility (GDF). 2020. Available from: http:/www.stoptb.org/ assets/documents/gdf/drugsupply/GDFMedicinesCatalog.pdf.

155. Blok, DJ. GPZL Reports on Research Priorities. Leprosy Review, 2020;90(3). Available from: https://zeroleprosy.org/wp-content/ uploads/2019/11/research-priorities.pdf.
Research and Reports in Tropical Medicine

\section{Publish your work in this journal}

Research and Reports in Tropical Medicine is an international, peerreviewed, open access journal publishing original research, case reports, editorials, reviews and commentaries on all areas of tropical medicine, including: Diseases and medicine in tropical regions; Entomology; Epidemiology; Health economics issues; Infectious disease; Laboratory science and new technology in tropical medicine;
Parasitology; Public health medicine/health care policy in tropical regions; and Microbiology. The manuscript management system is completely online and includes a very quick and fair peer-review system. Visit http://www.dovepress.com/testimonials.php to read real quotes from published authors. 